\title{
Inhibition of Piezo1 attenuates demyelination in the central nervous system
}

María Velasco-Estevez ${ }^{1,2}$, Kamal K. E. Gadalla ${ }^{3}$, Núria Liñan-Barba ${ }^{2}$, Stuart

$\mathrm{Cobb}^{3}$, Kumlesh K. Dev ${ }^{1, \S}$, Graham K. Sheridan ${ }^{2,4, \dagger}$

${ }^{1}$ Drug Development, School of Medicine, Trinity College Dublin, Dublin, Ireland.

${ }^{2}$ School of Pharmacy and Biomolecular Sciences, University of Brighton, Brighton, UK.

${ }^{3}$ Centre for Discovery Brain Sciences, University of Edinburgh, Edinburgh, UK.

${ }^{4}$ School of Life Sciences, Queen's Medical Centre, University of Nottingham, Nottingham UK.

\section{Correspondence should be addressed to:}

Dr. Graham Sheridan, School of Life Sciences, Queen's Medical Centre, University of Nottingham, Nottingham UK.

Email: Graham.Sheridan@nottingham.ac.uk

${ }^{\S}$ Co-corresponding author: Prof. Kumlesh Dev

Email:devk@tcd.ie

Running title: Piezo1 regulates CNS myelination.

Key Words: Cerebellum; GsMTx4; Mechanosensitive channels; Myelination; Organotypic slice cultures; Piezo1.

\section{Author contributions}

G.K.S. conceived the project. G.K.S., K.K.D. and S.C. designed the research; M.V.E., K.K.E.G., N.L.B. and G.K.S. performed the experiments; M.V.E. and G.K.S. analysed the data; all authors discussed the results; G.K.S., K.K.D. and M.V.E. wrote the manuscript with contributions from all authors.

DOI: $10.1002 /$ glia.23722 


\section{Financial Support}

This work was supported by an Irish Research Council PhD scholarship to M.V.E. [GOIPG/2015/2804] and a Rising Stars award to G.K.S from the University of Brighton. G.K.S is grateful for funding from the Royal Society [RG150155] and the Leverhulme Trust [RPG-2018-443].

\section{Acknowledgements}

The authors wish to thank Sinead O'Sullivan for valuable technical assistance in the early stages of the project.

\section{Conflict of Interest Statement}

The authors declare no conflicts of interest and no competing financial interests.

\section{Data availability}

The data that support the findings of this study are available from the corresponding author upon reasonable request.

\section{Abbreviations}

Analysis of variance

Bovine Serum Albumin

Central Nervous System

Days in vitro

Food and Drug Administration FDA;

Hank's balanced salt solution

Ionized calcium-binding adapter-1

Minimal Essential Medium

Myelin Basic Protein

Neural progenitor cell

Organotypic Cerebellar Slice

Postnatal day 10

Penicillin/Streptomycin

Proteolipoprotein

Polyvinylidene difluoride membrane

Reactive oxygen species

Sodium chloride

Standard Deviation

$\begin{array}{lll}\text { ANOVA; } & \text { Arbitrary fluorescence units } & \text { a.f.u } \\ \text { BSA; } & \text { Calcium } & \mathrm{Ca}^{2+} \\ \text { CNS; } & \text { Chi-squared } & \chi 2 \\ \text { DIV; } & \text { Duchenne Muscular Dystrophy } & \text { DMD } \\ & \text { Glial Fibrillary Acidic Protein } & \text { GFAP } \\ \text { HBSS; } & \text { Horse radish peroxidase } & \text { HRP } \\ \text { Iba-1; } & \text { Lysophosphatidylcholine } & \text { LPC } \\ \text { MEM; } & \text { Minutes } & \text { Min } \\ \text { MBP; } & \text { Myelin Oligodendrocyte GlycoproteinMOG } \\ \text { NPC; } & \text { Neurofilament H } & \text { NFH } \\ \text { OCS; } & \text { Oligodendrocyte precursors } & \text { OP } \\ \text { P10; } & \text { Paraformaldehyde } & \text { PFA } \\ \text { Pen/Strep; } & \text { Phosphate-buffered saline } & \text { PBS } \\ \text { PLP; } & \text { Psychosine } & \text { PSY } \\ \text { PVDF; } & \text { Radioimmunoprecipitation assay } & \text { RIPA } \\ \text { ROS; } & \text { Region of interest } & \text { ROI } \\ \text { NaCl; } & \text { Sodium dodecyl sulfate } & \text { SDS } \\ \text { SD; } & & \end{array}$




\title{
Main Points
}

- Piezo1 negatively regulates central nervous system myelination.

- Piezo1 antagonist, GsMTx4, enhances myelination and attenuates demyelination.

- GsMTx4 is neuroprotective and inhibits lysophosphatidylcholine-induced astrocyte toxicity.

\begin{abstract}
Piezo1 is a mechanosensitive ion channel that facilitates the translation of extracellular mechanical cues to intracellular molecular signalling cascades through a process termed, mechanotransduction. In the central nervous system (CNS), mechanicallygated ion channels are important regulators of neurodevelopmental processes such as axon guidance, neural stem cell differentiation, and myelination of axons by oligodendrocytes. Here, we present evidence that pharmacologically-mediated overactivation of Piezo1 channels negatively regulates CNS myelination. Moreover, we found that the peptide GsMTx4, an antagonist of mechanosensitive cation channels such as Piezo1, is neuroprotective and prevents chemically-induced demyelination. In contrast, the positive modulator of Piezo1 channel opening, Yoda-1, induces demyelination and neuronal damage. Using an ex vivo murine-derived organotypic cerebellar slice culture model, we demonstrate that GsMTx4 attenuates demyelination induced by the cytotoxic lipid, psychosine. Importantly, we confirmed the potential therapeutic effects of GsMTx4 peptide in vivo by co-administering it with lysophosphatidylcholine (LPC), via stereotactic injection, into the cerebral cortex of adult mice. GsMTx4 prevented both demyelination and neuronal damage usually caused by the intra-cortical injection of LPC in vivo; a well-characterised model of focal demyelination. GsMTx4 also attenuated both LPC-induced astrocyte toxicity and microglial reactivity within the lesion core. Overall, our data suggest that pharmacological activation of Piezol channels induces demyelination and that inhibition of mechanosensitive channels, using GsMTx4, may alleviate the secondary progressive neurodegeneration often present in the latter stages of demyelinating diseases.
\end{abstract}




\section{Introduction}

Piezo1 is a mechanically-gated cation channel and allows the influx of calcium ions $\left(\mathrm{Ca}^{2+}\right)$ into the cell when activated by mechanical cues (Gnanasambandam, Bae, Gottlieb, \& Sachs, 2015; Gnanasambandam et al., 2017). PIEZO proteins are highly conserved throughout evolution and play crucial roles in mechanosensation and mechanotransduction in multicellular organisms (Coste et al., 2012). In the vertebrate central nervous system (CNS), Piezo1 is expressed on neuronal cell membranes and regulates important developmental processes such as axon guidance. This was recently demonstrated in retinal ganglion neurons of developing Xenopus embryos (Koser et al., 2016). Moreover, elegant work by Pathak et al. (2014) showed that traction forcemediated activation of Piezo1 triggers $\mathrm{Ca}^{2+}$ influx and directs the lineage choice of neural stem cells toward a neuronal phenotype rather than astrocytic. More recently, we reported that reactive cortical astrocytes that engulf stiff amyloid plaques upregulate Piezo1 channels, as demonstrated in an ageing rat model of Alzheimer's disease (Velasco-Estevez et al., 2018). This suggests that glial mechanotransduction may be disrupted by amyloid plaque pathology. Moreover, the mechanoresponsive transcriptional coactivators, Yap and Taz, are targeted to the nucleus of stem cells following activation of Piezol (Pathak et al., 2014). However, before its important role in mechanosensation had been elucidated, Piezol was known by another name, FAM38A. McHugh et al. (2010) had previously shown how Piezo1 helps to maintain integrin-mediated cell adhesion in epithelial cells by recruiting the small GTPase, RRas, to the endoplasmic reticulum which, in turn, leads to $\mathrm{Ca}^{2+}$ release from internal stores, activation of calpain signalling (i.e. a $\mathrm{Ca}^{2+}$-dependent protease) and cleavage of talin (a protein that links membrane integrins and the actin cytoskeleton) (McHugh, Murdoch, Haslett, \& Sethi, 2012). Both integrin signalling and Yap/Taz nuclear localisation are key cellular events in the formation of CNS myelin (O'Meara, Michalski, \& Kothary, 2011; Shimizu et al., 2017). Here, we investigated the role of Piezo1 channel activity in both myelination and demyelination of the central nervous system.

Demyelinating disorders are defined by the progressive degeneration of the myelin sheath which is formed by specialised glial cells that envelop and insulate neuronal axons (Saab, Tzvetanova, \& Nave, 2013; Simons \& Nave, 2015). In the CNS, the close physical contact between oligodendrocytes and axons facilitates intimate neuron/glial 
crosstalk (Chang, Redmond, \& Chan, 2016) consisting of both biochemical and mechanotactic cell signalling (Jagielska et al., 2012). As such, both neuronal and oligodendroglial homeostasis are intimately linked; i.e. axonal injury often leads to demyelination, but the opposite is also true, in that myelin damage can trigger neurodegeneration (Hauser \& Oksenberg, 2006; Simons et al., 2014). Moreover, demyelinating disorders often display perturbations to axonal $\mathrm{Ca}^{2+}$ homeostasis caused by large influxes through membrane-bound ion channels or a sustained release of $\mathrm{Ca}^{2+}$ from intracellular stores (Stirling, Cummins, Wayne Chen, \& Stys, 2014), culminating in excitotoxicity. Elevated $\mathrm{Ca}^{2+}$ can also lead to the production of pathophysiological levels of reactive oxygen species (ROS) and oxidative damage which, in turn, activate apoptotic cascades (Azuma \& Shearer, 2008; Gorlach, Bertram, Hudecova, \& Krizanova, 2015; Wojda, Salinska, \& Kuznicki, 2008). Consequently, abnormally high $\mathrm{Ca}^{2+}$ influx can cause the destabilisation and unravelling of axonal cytoskeletal transport machinery, ultimately causing irreversible neurodegeneration (Barsukova, Forte, \& Bourdette, 2012; Frati et al., 2017). As such, neuronal damage often exacerbates CNS myelin breakdown (Baloh, 2008). Therefore, therapeutics that can inhibit excessive $\mathrm{Ca}^{2+}$ influx or pathophysiological calcium-induced calcium release from intracellular stores may hold therapeutic potential in demyelinating diseases. Here, we present evidence that pharmacological blockade of mechanosensitive Piezo1 channels prevents axonal and myelin damage in the CNS, both in vitro and in vivo. 


\section{Materials and Methods}

\section{Ethics statement:}

All experiments involving animals and Schedule 1 protocols used to obtain brain tissue were approved by the Animal Welfare and Ethical Review Bodies (AWERB committees) of the University of Brighton and the University of Glasgow, as well as the Animal Research Ethics Committee (AREC) in Trinity College Dublin. Surgical procedures were carried out under UK Home Office licence-approved protocols. This study was conducted in accordance with the principles of the Basel Declaration and adhered to the legislation detailed in the UK Animals (Scientific Procedures) Act 1986 Amendment Regulations (SI 2012/3039). All efforts were taken to maximise animal welfare conditions and to reduce the number of animals used in accordance with the European Communities Council Directive of September 20 $0^{\text {th }}, 2010$ (2010/63/EU).

\section{Reagents:}

GsMTx4 (also known as AT-300) has been designated an orphan drug by the US food and drug administration (FDA) and counteracts cellular $\mathrm{Ca}^{2+}$ dysregulations in preclinical models of muscular dystrophy (Ward, Sachs, Bush, \& Suchyna, 2018). The 34-amino acid peptide (Alomone labs, STG-100) is a member of the Inhibitory Cysteine Knot family with six cysteines, is a non-toxic component of tarantula venom, and functions as a negative allosteric modulator of mechanoreceptors (Suchyna et al., 2000). The D and L enantiomers of GsMTx4 have almost identical activity (Wang, Ma, Sachs, Li, \& Suchyna, 2016) which suggests that its mechanism is not reliant on direct stereo-chemical interactions (Gnanasambandam et al., 2017). Instead, it has high affinity for lipid bilayers and its ability to partition into membranes and inhibit mechanosensitive ion channel opening appears key to its mechanism of action as a gating modifier of Piezo1 (Gottlieb, Suchyna, \& Sachs, 2007).

Yoda-1 (Tocris, 5586) is a synthetic small molecule that specifically activates Piezo1 at micromolar concentrations (Syeda et al., 2015; Evans et al., 2018) by interacting with the agonist transduction motif of Piezo1 subunits, thus enhancing channel opening-time (Lacroix, Botello-Smith, \& Luo, 2018). In contrast to GsMTx4, Yoda-1 may interact directly with Piezo1 domains rather than modifying the lipid environment around the ion channel (Syeda et al., 2015). 
Galactosylsphingosine or psychosine (Santa Cruz Biotechnology, sc-202781) is a cationic lysosphingolipid that accumulates in the brains of patients with Krabbe disease (Giri et al., 2002) and causes oligodendrocyte cell death and demyelination (Misslin, Velasco-Estevez, Albert, O'Sullivan, \& Dev, 2017; C. O'Sullivan \& Dev, 2015).

Lysophosphatidylcholine (LPC; Sigma, \#L4129) is an endogenous lysophospholipid that disrupts myelin-associated lipids (Plemel et al., 2018) leading to focal demyelination. It has been shown that the LPC-induced demyelination of axons requires an increase in intracellular $\mathrm{Ca}^{2+}$ levels (Fu, Wang, Huff, Shi, \& Cheng, 2007).

\section{Organotypic cerebellar slice culture model}

Organotypic cerebellar slice (OCS) cultures were prepared from postnatal day 10 (P10) C57BL/6 mice. Mice were sacrificed by decapitation, the skull removed, and the cerebellum separated from the hindbrain. The cerebellum was sliced into $400 \mu \mathrm{m}$ parasagittal sections using a Mcllwain tissue chopper. The tissue was placed into a petri dish with Opti-MEM (Gibco, 31985) and separated into individual slices under a dissecting microscope. Five slices per organotypic insert (Millicell, PICMORG50) were cultured, with the first medium change taking place $24 \mathrm{hr}$ after the dissection. For the first four days, slices were cultured in serum-based medium composed of $50 \%$ Opti-MEM, 25\% HBSS (Gibco, 14025-050), 25\% heat-inactivated horse serum (Gibco, 26050-088), supplemented with 2 mM GlutaMAX ${ }^{\text {TM }}$ (Gibco, 35050061), 28 mM D-Glucose (Sigma, G8769), 10 mM HEPES (Gibco, 15630-056), 100 units/mL penicillin and $100 \mu \mathrm{g} / \mathrm{mL}$ streptomycin (Pen/Strep) (Sigma, P4333). On day 4 in vitro, the medium was changed to a serum-free composition containing $98 \%$ Neurobasal-A (Gibco, 10888-022), 2\% B-27 (Gibco, 17504-044), 28 mM D-Glucose, 2 mM GlutaMAX ${ }^{\mathrm{TM}}, 10 \mathrm{mM}$ HEPES, 100 units/mL penicillin and $100 \mu \mathrm{g} / \mathrm{mL}$ streptomycin. OCS cultures were replenished with fresh serum-free medium changes on days 7 and 10 and drug treatments began on day 12 in vitro.

\section{Immunofluorescence of slice cultures}

OCS cultures were fixed using increasing concentrations of paraformaldehyde (PFA; $1 \%, 2 \%, 3 \%$ and $4 \%$ ) for 5 min each. Blocking and permeabilization was performed 
at $4^{\circ} \mathrm{C}$ for $18 \mathrm{hr}$ in a phosphate-buffered saline (PBS) solution containing $10 \% \mathrm{BSA}+$ $0.5 \%$ Triton X-100. Primary antibodies were diluted in $2 \% \mathrm{BSA}+0.1 \%$ Triton $\mathrm{X}-100$ and incubated for $48 \mathrm{hr}$ at $4^{\circ} \mathrm{C}$. Slices were washed with PBS $+0.1 \%$ Triton X-100 buffer three times for $10 \mathrm{~min}$. Incubation with secondary antibodies was performed at $4^{\circ} \mathrm{C}$ for $18 \mathrm{hr}$. Slices were washed again and mounted on microscope slides using ProLong $^{\circledR}$ Gold antifade reagent (ThermoFisher Scientific, P36934). Primary antibodies included: goat anti-PIEZO1 (N-15; Santa Cruz, sc-164319, RRID: 32 AB_10842990; 1/500 dilution); rabbit anti-myelin basic protein (MBP) (Abcam, ab40390; RRID: AB_1141521; 1/1000 dilution); mouse monoclonal antiproteolipoprotein (PLP) (Millipore, MAB388; RRID: AB_177623; 1/1000 dilution); mouse monoclonal anti-myelin oligodendrocyte glycoprotein (MOG) (Millipore, MAB5680; RRID: AB_1587278; 1/1000 dilution); chicken anti-neurofilament heavy (NFH) (Millipore, AB5539; RRID: AB_177520; 1/1000 dilution); mouse monoclonal anti-vimentin (Santa Cruz, sc-373717; RRID: AB_10917747; 1/1000 dilution); rabbit anti-ionized calcium-binding adapter molecule 1 (Iba1) (Wako, 019-19741; RRID: AB_839504; 1/1000 dilution); chicken polyclonal anti-GFAP (Abcam, ab4674; RRID: AB_304558; dilution 1/1,000) and mouse monoclonal anti-SMI-32 (Millipore, NE1023; RRID: AB_2043449; 1/1000 dilution). Secondary antibodies used included: donkey anti-goat Alexa Fluor 488 (Abcam, ab150133; RRID: AB_2687506; 1/2000 dilution); goat anti-rabbit Alexa Fluor 488 (ThermoFisher Scientific, A11008; RRID: AB_143165; $1 / 2000$ dilution), donkey anti-rabbit IgG CFTM 555 (Sigma, SAB4600061; 1/2000 dilution), goat anti-mouse DyLight 549 (Jackson ImmunoResearch, 115-506-068; 1/2000 dilution), donkey anti-chicken IgY CFTM 633 (Sigma, SAB4600127; 1/2000 dilution), and goat anti-chicken IgY Alexa Fluor 633 (ThermoFisher Scientific, A21103; RRID: AB_2535756; 1/2000 dilution).

\section{Microscopy and image analysis of slice cultures}

Immunofluorescence images of OCS cultures were captured at $40 \times$ magnification using a Leica SP8 confocal microscope. For each experiment $(n \geq 5)$, there were 5 slices per treatment group and 5-6 fluorescence images were captured per slice. The areas of the cerebellum captured were kept consistent between treatment groups. The images were exported as 8-bit tif files for analysis using the software package FIJI. To quantify fluorescence intensity, 10 regions of interest (ROI) were manually selected from each image and the average fluorescence intensity within each ROI was 
calculated. Intensity values were normalised to the average control value for each protein of interest. To analyse the expression of SMI-32 in the white matter tracts, the software package Imaris ${ }^{\circledR} 9$ was used. Briefly, an ROI containing predominantly white matter tracts was manually selected in each image. The proportion of this white matter area that stained positive for SMI-32 immunoreactivity (termed SMI-32 surface area) was quantified.

\section{Western blot}

Protein samples were prepared from OCS cultures by homogenising slices in radioimmunoprecipitation assay (RIPA) buffer containing $150 \mathrm{mM}$ sodium chloride $(\mathrm{NaCl}), 1 \%$ Triton $\mathrm{X}-100,0.1 \%$ sodium dodecyl sulfate (SDS) and $50 \mathrm{mM}$ Tris $\mathrm{pH}$ 8.0. Samples were sonicated three times for $10 \mathrm{sec}$ at $20 \%$ amplitude using a vibracell VCX 130 (Sonics, USA). Samples were mixed in 1:1 ratio with Laemmli sample buffer 2X (BioRad, 161-0737) and boiled at $95^{\circ} \mathrm{C}$ for $5 \mathrm{~min}$. Samples were run in $15 \%$ acrylamide/bisacrylamide gels (Applichem Panreac, A1672), loading $6 \mu \mathrm{g}$ of total protein per well. The gels were run at a constant voltage of $120 \mathrm{~V}$ and wet transfer was performed using polyvinylidene difluoride membrane (PVDF; Millipore, IPVH00010) at constant $75 \mathrm{~mA}$ for $75 \mathrm{~min}$. The membranes were blocked in $5 \%$ bovine serum albumin (BSA; Santa Cruz, sc-2323) in PBS $+0.05 \%$ Tween for $1 \mathrm{hr}$ at $22^{\circ} \mathrm{C}$. Membranes were then incubated with primary antibodies for $18 \mathrm{hr}$ at $4^{\circ} \mathrm{C}$. To remove excess primary antibody, membranes were washed in PBS and then incubated with secondary antibodies for $1.5 \mathrm{~h}$ at $22^{\circ} \mathrm{C}$. Membranes were developed after several washes using chemiluminescent HRP substrate (Millipore, WBKLS0500).

\section{Stereotactic surgery}

For stereotactic injection of compounds into the left and right cerebral cortex, 10 male C57BL/6 mice (9-10 weeks old, 20-30 g in weight; Envigo, UK) were randomly divided into three groups: 1) LPC 0.1\% (left) vs PBS (right; $\mathrm{n}=2$ ); 2) LPC $0.1 \%$ (left) vs LPC $0.1 \%+3 \mu \mathrm{M}$ GsMTx4 (right; $\mathrm{n}=4$ ); and 3) PBS (left) vs LPC $0.1 \%+3 \mu \mathrm{M}$ GsMTx4 (right; $\mathrm{n}=4$ ). Mice were injected intraperitoneally with $1.25 \%$ Avertin (tribromoethanol, $20 \mu \mathrm{L} / \mathrm{g}$ body weight) in PBS prior to $3 \%$ isoflurane inhalation anaesthesia, followed by shaving the skull from between the eyes to the base of the neck. Mice were positioned into a small animal stereotactic frame $\left(\mathrm{KOPF}^{\circledR}\right.$; California, USA), Lacri-Lube eye ointment was applied to prevent dryness of the eyes, and 
anaesthesia was maintained using $1.5-2 \%$ isoflurane during the procedure. The skin over the skull was sterilized using iodine and a midline incision was made to expose skull bone. To minimise post-operative pain, $1 \%$ lidocaine was applied on the skull for 5 min prior to softening the skull bone using a fine drill at the following injection coordinates; $1 \mathrm{~mm}$ rostral to bregma, $1 \mathrm{~mm}$ lateral to the midline, and a depth of 1.5 $\mathrm{mm}$ from the surface of the brain (Fig. 9A, B). The sites of injection corresponded to a region of the primary somatosensory cortex, proximal to the posterior motor cortex (Fig. 9C - E). Injections were performed using a Hamilton ${ }^{\mathrm{TM}}$ Neuros syringe 1710RN (Esslab, 7656-01) and Hamilton ${ }^{\mathrm{TM}}$ needle RN 336-A (Esslab, 65461-01). The needle was introduced slowly into the brain at a speed of $0.5 \mathrm{~mm} / \mathrm{min}$. The compounds $(1 \mu \mathrm{L})$ were injected over a 6 min period and the needle was kept in place for an additional 5 $\mathrm{min}$, then withdrawn gradually $(0.5 \mathrm{~mm} / \mathrm{min})$ to minimize backflow of the injected substances. The skin was sutured with Vicryl ${ }^{\circledR}$ 6-0 taperpoint curve needle (Ethicon, W9981). Mice recovered post-operatively in warm air chambers with easy access to water and a soft diet for $24 \mathrm{hr}$ and were then transferred to ordinary cages until the end of the experiment. Four days post-surgery, mice were injected with a terminal dose of pentobarbital and were transcardially-perfused with 4\% PFA in $0.1 \mathrm{M}$ PBS. The whole brain was dissected out and placed in 4\% PFA for an additional $4 \mathrm{hr}$ at $4{ }^{\circ} \mathrm{C}$ and then submerged in 30\% sucrose in $0.1 \mathrm{M}$ PBS for one week. Brains were then embedded in OCT, snap-frozen in liquid nitrogen and stored at $-80^{\circ} \mathrm{C}$ until cryosectioning was performed.

\section{Tissue cryosectioning and immunofluorescence}

Mouse brains were cryosectioned using a Leica cryostat at a thickness of $12 \mu \mathrm{m}$ and sections were placed onto SuperFrost ${ }^{\mathrm{TM}}$ Plus adhesion slides (Fisher, 10149870) which were allowed to air-dry for $30 \mathrm{~min}$ and then stored at $-80^{\circ} \mathrm{C}$ until required. For immunofluorescence, microscope slides were removed from the $-80^{\circ} \mathrm{C}$ freezer and allowed to air-dry at $22^{\circ} \mathrm{C}$ for 20 min prior to rehydration in PBS. For myelin basic protein (MBP) and neurofilament $\mathrm{H}(\mathrm{NFH})$ staining, antigen retrieval was performed in $95^{\circ} \mathrm{C}$ Tris-EDTA buffer (10 mM Tris-base, 0.5M EDTA pH 9.0, 0.05\% Tween-20) for $30 \mathrm{~min}$. All slides were permeabilized in $0.2 \%$ Triton-X in PBS for $30 \mathrm{~min}$, followed by three PBS washes. Next, sections were blocked with 10\% BSA in PBS for $3 \mathrm{hr}$ at $22^{\circ} \mathrm{C}$. Blocking solution was removed and brain sections were incubated with primary antibodies diluted in $2 \% \mathrm{BSA} / \mathrm{PBS}$ and kept at $4^{\circ} \mathrm{C}$ for $18 \mathrm{hr}$. Slides were then 
washed five times in PBS and incubated with secondary antibodies diluted in 2\% BSA/PBS for $18 \mathrm{hr}$ at $4^{\circ} \mathrm{C}$ and protected from light. Finally, slides were washed and mounted with coverslips using ProLong ${ }^{\circledR}$ Gold antifade reagent. Primary antibodies included: rabbit anti-MBP (Abcam, ab40390; RRID: AB_1141521; 1/1000 dilution); chicken anti-NFH (Millipore, AB5539; RRID: AB_177520; 1/1000 dilution); chicken anti-glial fibrillary acidic protein (GFAP) (Abcam, ab4674; RRID: AB_304558; 1/1000 dilution), and goat anti-Iba1 (Novus Bio, NB100-1028; RRID: AB_521594; 1/400 dilution). Secondary antibodies included: donkey anti-rabbit Alexa Fluor 488 (Abcam, ab150065; 1/2000 dilution), donkey anti-mouse $\mathrm{CF}^{\mathrm{TM}} 555$ (Sigma, SAB4600060; 1/2000 dilution), donkey anti-chicken IgY CF ${ }^{\mathrm{TM}} 633$ (Sigma, SAB4600127; 1/2000 dilution), and donkey anti-goat Alexa Fluor 488 (Abcam, ab150133; 1/2000 dilution).

\section{Microscopy and image analysis of mouse brain sections}

Immunofluorescence images of the left and right hemispheres of each mouse brain section were captured at $40 \times$ magnification using a Leica SP5 confocal microscope. There were 3 groups of mice, as described above, and 2-3 brain sections were analysed per mouse. The images were exported as 8-bit tif files for analysis using the software package FIJI. To quantify fluorescence intensity, 10 ROI were manually selected from each image and the average fluorescence intensity within each ROI was calculated. Fluorescence intensity values are displayed as arbitrary fluorescence units (a.f.u.). For cell count and surface area analysis, FIJI's particle analyser tool was employed. Briefly, images were converted from 8-bit to binary and a value of 20 pixels was set as the minimum particle size. A mask of the particles detected was generated to check the accuracy of the detection method. A numbered list of particles displaying the fluorescence intensity and the area of each were analysed. To analyse NFH and MBP co-localisation, the Manders split coefficient was calculated using FIJI software, which is a value between 0 and 1 and is proportional to the amount of NFH positive pixels that overlap with MBP positive pixels. Demyelination was assessed by calculating a decrease in the proportion of NFH staining co-localised with MBP. The accumulation of myelin debris was quantified by calculating an increase in the proportion of MBP staining which did not co-localise with NFH-positive axons. 


\section{Statistical analysis}

For organotypic experiments, there were five technical replicates (i.e., slices) per treatment group and each experiment was repeated between $n=5$ and $n=11$ times, as indicated in the appropriate figure legend. Separate experiments were counted as slices that were extracted from different brain tissue from different mouse litters on different experimental days. In this instance, " $n$ " refers to the number of independent organotypic slice culture preparations performed on different experimental days. For each slice culture, 25 healthy slices were obtained from the cerebellum of five mouse littermates and were randomly separated into the five different treatment groups, as described in Figure 2f. Therefore, if an experiment was repeated $n=5$, a total of 25 cerebellar slices were stained and imaged for each treatment group. For in vivo animal experiments, " $n$ " refers to the number of mice per experimental group. All post hoc statistical analysis was performed using GraphPad® Prism 7 (RRID:SCR_002798). Each dataset was tested for normality using column statistics and the D'AgostinoPearson omnibus test prior to any other statistical analysis being performed. For Western blot assays, repeated measures analysis of variance (ANOVA) tests were performed because data in every experiment were matched. Data are presented as the mean $\pm S D$. Holm-Sidak multiple comparisons post hoc tests were run in conjunction with one-way ANOVAs and all groups were compared with one another. To analyze changes in the fluorescence intensities of cell markers and of SMI-32 expression in OCS cultures, repeated measures one-way ANOVAs were performed, followed by Holm-Sidak multiple comparisons post hoc tests. Data are presented as the mean $\pm S E M$. Changes in the fluorescence intensity of cell markers, cell count, the surface area of astrocytes and microglia in vivo, the number of astrocyte branches and their average length were analyzed using two-way ANOVAs with Holm-Sidak multiple comparisons post hoc tests, comparing the left versus right hemispheres for each treatment group. Finally, the analysis of proportions for amoeboid, ramified, and intermediate-shaped microglia was carried out using chi-squared $\left(\chi^{2}\right)$ tests in conjunction with the Bonferroni correction for multiple comparisons. In all cases, a $p$ value of $<.05$ was deemed to be statistically significant. 


\section{Results}

\section{Piezo1 is expressed by neurons in the mouse brain}

We have recently characterised expression of the mechanosensitive channel, Piezo1, in young and adult rat brain (Velasco-Estevez et al., 2018). To visualise expression of Piezo1 in the mouse brain, horizontal sections were cut from 5-week old C57BL/6 mice and immunofluorescently stained for Piezo1, the myelin marker PLP, and the axonal marker NFH. Similar to the rat (Velasco-Estevez et al., 2018), Piezo1 localised to myelinated axonal pathways of the mouse brain, including the corpus callosum and cerebellar arbor vitae (Fig. 1A - D). To determine if Piezo1 is expressed by neurons and/or mature oligodendrocytes, high magnification images of a more sparsely myelinated area of the frontal cortex were captured (Fig. 1E - J). Notably, Piezo1 did not co-localise with PLP (Fig. 1G) but instead with NFH (Fig. 1J), suggesting that Piezo1 is expressed predominantly by neurons in the mouse frontal cortex. Since the function of neuronal Piezo1 in the postnatal and adult brain is unknown, we next used organotypic cerebellar slice cultures, a highly myelinated brain region, to investigate if Piezo1 plays a role in CNS myelination.

\section{Blocking mechanosensitive ion channels enhances myelination and prevents psychosine-induced demyelination}

To confirm Piezo1 expression in organotypic cerebellar slices, the tissue was fixed and triple-immunolabelled for Piezol (Fig. 2A), PLP (Fig. 2B), and NFH (Fig. 2C). Similar to its expression pattern in vivo, Piezo1 mainly localised to myelinated neurons in cerebellar slices (Fig. 2D), although the expression pattern was more diffuse than in fresh frozen sections from 5-week old mice. After 12 days in vitro, organotypic cerebellar slices were treated with a specific activator of Piezo1, i.e. Yoda-1 (10 $\mu \mathrm{M}$ for $48 \mathrm{hr}$ ), or a peptide inhibitor of Piezo1, i.e. GsMTx4 (500 nM for $48 \mathrm{hr}$ ), in order to investigate how activating or blocking Piezo1 affects myelination. Slices were also treated with the demyelinating agent psychosine $(100 \mathrm{nM}$ for $18 \mathrm{hr}+30 \mathrm{hr}$ of fresh medium) in the presence or absence of GsMTx4 (500 nM for $48 \mathrm{hr}$ ) and myelination was assessed by PLP fluorescence intensity (Fig. 2E). A summary of the pharmacological treatment regime for slices is illustrated in Fig. 2F. Exposure to Yoda1 or psychosine caused similar levels of demyelination (Fig. $2 \mathrm{G}$ ). We have previously reported that psychosine induces demyelination of organotypic cerebellar slices 
(Misslin et al., 2017; C. O'Sullivan \& Dev, 2015) and have used this system as an ex vivo model of the dysmyelination that occurs in Krabbe disease (Giri et al., 2002). Importantly, blocking Piezo1 channels with GsMTx4 prevented psychosine-mediated demyelination. Moreover, GsMTx4 alone enhanced myelination over and above control levels, measured as an increase in PLP fluorescence intensity. This suggests that over-activation of neuronal Piezol disrupts myelination, whilst inhibition may promote myelin formation. These results were confirmed by measuring the effects of Yoda-1 and GsMTx4 on the levels of MOG (Fig. 3A, C) and MBP (Fig. 3B, D) in cerebellar slice cultures, using the same protocol described in Fig. 2F. Furthermore, we quantified the stripping of myelin from axons by calculating the proportion of NFH staining co-localised with the myelin marker, MBP. Neither Yoda-1 nor psychosine caused significant stripping of the myelin sheath from the axon, in vitro (Fig. 3E, F). However, both Yoda-1 and psychosine caused a significant accumulation of 'myelin debris', defined as an increase in the proportion of MBP staining that did not colocalise with NFH+ axons (Fig. 3G). GsMTx4 prevented the increase in myelin debris accumulation caused by psychosine. Next, to measure the relative abundances of myelin-associated proteins, MOG and MBP, in the cerebellar slice as a whole; protein samples were prepared from cultures treated using the same protocol described in Fig. 2F. Western blot was performed to measure changes in MOG (Fig. 4A) and MBP (Fig. 4B) protein levels with respect to total actin expression. There were no statistically significant differences in MOG or MBP protein expression between treatment groups. However, the pattern of expression did somewhat mimic the immunofluorescence results, suggesting that Yoda-1 is detrimental to myelin formation and GsMTx4 may rescue psychosine-induced demyelination.

\section{Demyelination-induced axonal damage is attenuated by GsMTx4}

Next, we examined whether blocking Piezo1 channels prevents the axonal damage that often accompanies demyelination. Slices were once again treated using the same protocol described in Fig. $2 \mathrm{~F}$ and then fixed and stained for SMI-32, a nonphosphorylated epitope of NFH (Louis et al., 2012) and a marker of axonal damage and demyelination (Linder et al., 2009; Misslin et al., 2017; S. A. O'Sullivan, VelascoEstevez, \& Dev, 2017). Organotypic cerebellar slices treated with the Piezo1 activator, Yoda-1 (10 $\mu \mathrm{M}$ for $48 \mathrm{hr}$ ), displayed an increase in SMI-32 expression in the axons of cerebellar Purkinje neurons (Fig. 5A, B). As previously reported, psychosine also 
significantly upregulated SMI-32 expression levels. Importantly, co-treatment with GsMTx4 prevented psychosine-induced axonal damage (Fig. 5A, B). We also fixed and immunofluorescently-labelled slices for phosphorylated neurofilament H (NFH), known to be important in the maintenance of axonal calibre and structural integrity of neuronal axons (Rudrabhatla, 2014). Neither psychosine nor Yoda-1 had any effect on NFH expression (Fig. 6A). In contrast, treatment with the inhibitor GsMTx4 (500 nM for $48 \mathrm{hr}$ ) significantly increased the levels of NFH (Fig. 6A), suggesting a neuroprotective effect on mature Purkinje axons. However, GsMTx4 did not display the same protective actions for neural progenitor cells (NPCs) or immature astroglia, as assessed by Vimentin expression (Fig. 6B). Yoda-1 did not cause NPC toxicity. However, psychosine caused a decrease in Vimentin expression which was not rescued by co-treatment with GsMTx4, suggesting that NPCs and immature astroglia may not express as many Piezo1 channels as mature neurons.

\section{GsMTx4 does not prevent psychosine-mediated astrocyte toxicity in vitro}

We next investigated the effects of Yoda-1 and GsMTx4 on microglial reactivity in cerebellar slice cultures. Neither activating nor blocking Piezol channels had any significant effects on microglial expression of Ibal (Fig. 7A). It must be noted, however, that Iba1 is not a specific marker of altered microglia reactivity. Similarly, neither Yoda-1 nor GsMTx4 had any significant effects on astrocyte reactivity, assessed by GFAP fluorescence intensity (Fig. 7B). Psychosine, on the other hand, caused a decrease in GFAP expression which was not attenuated by co-treatment with GsMTx4 (Fig. 7B). The effects of psychosine, GsMTx4 and Yoda-1 on neural progenitor cells/ immature astroglia (Vimentin expression), microglia (Iba1 expression) and mature astrocytes (GFAP expression) were corroborated using Western blot (Fig. 8A-C). Psychosine caused both NPC and astroglial toxicity which was not rescued by blocking Piezo1 channels using GsMTx4. There were no significant changes in microglial Ibal expression in any of the treatment groups. This suggests that Piezo1 is predominantly expressed by mature neurons as opposed to glial cell types in the mouse cerebellum.

GsMTx4 attenuates microglia and astrocyte reactivity caused by LPCinduced demyelination in vivo

To further investigate the potential therapeutic properties of GsMTx4, we employed 
an in vivo model of toxin-induced focal demyelination of cortical brain tissue (Irvine \& Blakemore, 2008; Plemel et al., 2018). In this case, we used the toxin LPC (Plemel et al., 2018) to determine both the protective effects of GsMTx4 in vivo and to investigate if these effects were specific to psychosine. In this set of experiments, mice were injected in the left and right cerebral hemispheres (Fig. 9A, B) with either: PBS, LPC $(0.1 \%)$, or LPC + GsMTx4 (3 $\mu \mathrm{M})($ Fig. 9C - E). Mice were sacrificed four days post-injection and the brains were processed for immunofluorescence. Unlike psychosine, LPC induced a potent increase in microglial reactivity (measured by enhanced Iba1 fluorescence intensity) and microglial cell numbers within the lesion site (Fig. 9F). The surface area of individual microglial cells also increased, and this was reflected by a change in cell shape from a quiescent ramified shape (PBS) to a more amoeboid shape following LPC injection (Fig. 9G). Co-injection of GsMTx4 with LPC prevented this enhanced microglial reaction (Fig. 9H). The number of microglial cells in the lesion core were reduced following LPC + GsMTx4 coadministration and the cells present largely remained in the ramified state (Fig. 9I). These results were confirmed by comparing animals injected with PBS in the left cerebral hemisphere and with LPC + GsMTx4 in the right (Fig. 9J). There were no differences in microglial numbers, Iba1 expression or microglial morphology between PBS and LPC + GsMTx4 treated hemispheres (Fig. 9J, K).

Next, the effects of LPC on astrocyte reactivity was assessed in the same mice. LPC induced a measurable decrease in the number of GFAP + cells and GFAP fluorescence intensity within the demyelinated lesion site (Fig. 10A). Moreover, astrocyte morphology was altered, measured as a decrease in the average surface area and a decrease in the number of branches of astrocytes present within the lesion core (Fig. 10A). Importantly, co-injection of GsMTx4 prevented LPC-mediated astrocyte toxicity (Fig. 10B) by attenuating the decrease in GFAP+ cells and GFAP fluorescence intensity seen with LPC alone. Of note, when compared to PBS treated hemispheres, LPC + GsMTx 4 caused an increase in the average surface area and a decrease in the branch length of GFAP+ astrocytes (Fig. 10C), without affecting the number of branches per astrocyte. This suggests that GsMTx4 can rescue astrocyte cell death and may facilitate astrocyte hypertrophy. If the astrocyte hypertrophy subsides and does not become a chronic glial scar, this may accelerate tissue recovery and re-myelination following CNS injury (Ishibashi et al., 2006; Sofroniew, 2009; Sofroniew \& Vinters, 2010; Su et al., 2009). 
GsMTx4 attenuates LPC-induced demyelination in the cerebral cortex in vivo

Lastly, we investigated the effects of GsMTx4 on LPC-induced demyelination in vivo. Injection of LPC into the cortex caused significant focal demyelination compared to vehicle control PBS (Fig. 11A - C). This was measured by, 1) a decrease in MBP fluorescence intensity, 2) a decrease in the proportion of NFH-positive axons colocalised with MBP protein (myelin stripping) and, 3) an increase in the proportion of MBP which was not co-localised with NFH (i.e. myelin debris). Importantly, coinjection of GsMTx4 with LPC prevented demyelination (Fig. 11D - F). This was confirmed by comparing mice that had been injected with PBS in the left hemisphere and LPC + GsMTx4 in the right. Here, MBP expression matched that of vehicle controls (Fig. 11G - I). LPC injection also caused damage to cortical axons, measured as a fragmented pattern and a decrease in the intensity of NFH staining within the lesion core (Fig. 11A, B). The most promising result found, from a translational neurology viewpoint, was that co-injecting GsMTx4 completely prevented the neurodegeneration caused by LPC (Fig. 11D, E). This indicates that blocking Piezo1 channels with GsMTx4 peptide may prove to be a novel therapeutic strategy for treating demyelinating diseases of the CNS. 


\section{Discussion}

Chronic and progressive demyelination of CNS neurons often leads to irreversible axonopathy and neurodegeneration (Castelvetri et al., 2011). Therefore, identifying compounds that show both neuroprotective and myelin promoting properties may prove valuable in the development of novel therapeutics for demyelinating diseases. Here, we report that the mechanosensitive ion channel blocker, GsMTx4, can prevent both psychosine-induced demyelination of organotypic slice cultures and LPCmediated demyelination of cortical neurons in vivo. It is not yet known if GsMTx4 exerts a direct beneficial effect on oligodendrocytes or an indirect protective mechanism of action on myelinated axons. Indeed, both scenarios are possible. What the data presented here do support, is that CNS neurons appear to express higher levels of Piezo1 than mature oligodendrocytes (Fig. 1). It will be important to confirm this finding using different molecular approaches because this will help to determine how exposure of cerebellar slice cultures to Yoda-1 triggers demyelination (Figs. 2, 3, 4) and how overactivation of Piezol leads to axonal damage in Purkinje neurons (Fig. 5). On the contrary, blocking Piezo1 channels with GsMTx4 peptide increased the levels of phosphorylated NFH (Fig. 6). Phosphorylation of NFH has been shown to enhance axonal calibre which, in turn, promotes myelination (Hsieh et al., 1994; Yuan et al., 2017). This neuroprotective property of GsMTx4 may increase the likelihood that axons will become myelinated by neighbouring oligodendrocyte precursor cells (OPCs).

We have recently found that Yoda- 1 increases $\mathrm{Ca}^{2+}$ influx into primary mouse astrocytes and triggers $\mathrm{Ca}^{2+}$ release from intracellular stores, particularly under inflammatory conditions (i.e. after $24 \mathrm{hr}$ exposure to $100 \mathrm{ng} / \mathrm{mL}$ lipopolysaccharide) (Velasco-Estevez et al. 2019). Therefore, we hypothesise that overactivation of neuronal Piezo1 in cerebellar slice cultures may trigger excitotoxic levels of calcium entry into CNS axons which, in turn, could cause the release $\mathrm{Ca}^{2+}$ from intracellular stores and activation of the $\mathrm{Ca}^{2+}$ dependent protease, calpain, which is known to be involved in LPC-mediated demyelination (Fu et al., 2007). Whilst this is speculative at present, the data presented here suggests that blocking Piezol channels in vivo protects astrocytes from LPC-induced toxicity (Fig. 10). Therefore, by preventing astrocyte cell death and the accumulation of myelin debris that often accompanies 
toxin-induced demyelination (Kocur et al., 2015; Ousman \& David, 2000), GsMTx4 also attenuates microglial reactivity within the core of CNS lesions (Fig. 9).

\section{Regulation of CNS myelination by GsMTx4}

Demyelinating diseases display underlying dysregulations to neuronal $\mathrm{Ca}^{2+}$ homeostasis which lead to eventual axonopathy and neurodegeneration (Galbiati et al., 2009; Zundorf \& Reiser, 2011). By correcting intracellular $\mathrm{Ca}^{2+}$ perturbations, it may be possible to prolong neuronal function, delay disease progression, and promote the innate remyelination capacity of the CNS (Franklin, ffrench-Constant, Edgar, \& Smith, 2012). Based on elegant work by various groups who have characterised the functions of Piezo1 channels in different tissues (Coste et al., 2010; Koser et al., 2016; McHugh et al., 2010; Pathak et al., 2014; Poole, Herget, Lapatsina, Ngo, \& Lewin, 2014), and investigated the effects of GsMTx4 on distinct cell types (Blumenthal, Hermanson, Heimrich, \& Shastri, 2014; Gottlieb \& Sachs, 2012; Gottlieb et al., 2007; JacquesFricke, Seow, Gottlieb, Sachs, \& Gomez, 2006); we propose that the neuroprotective actions of GsMTx4 reported here may rely on its ability to regulate intracellular calcium levels (Fig. 12). In support of this hypothesis, GsMTx4 has shown early promise as a therapy for Duchenne muscular dystrophy (DMD) (Yeung et al., 2005), which is characterised by the accumulation of excessive levels of intracellular $\mathrm{Ca}^{2+}$ concentrations in muscle cells that lack a proper functioning dystrophin protein (Allen, Whitehead, \& Froehner, 2016). DMD-affected muscle cells are subjected to enhanced $\mathrm{Ca}^{2+}$ entry through mechanosensitive channels and GsMTx4 inhibits this excessive $\mathrm{Ca}^{2+}$ influx, thus limiting muscle degeneration. It is also well established that GsMTx4 blocks $\mathrm{Ca}^{2+}$ entry through Piezo1 channels (Ilkan et al., 2017) and acts as a negative modulator of channel opening probability for mechanosensitive channels like the canonical transient receptor potential channels, TRPC1 and TRPC6 (Gottlieb et al., 2007). Several voltage-gated sodium and potassium channel currents are also blocked by GsMTx4 at higher concentrations (Redaelli et al., 2010), in contrast to TRPA1 channels which become more likely to open in the presence of GsMTx4 (Hill \& Schaefer, 2007). Importantly, TRP channels and voltage-gated sodium and potassium channels are also expressed by oligodendrocytes (Berret et al., 2017; Paez et al., 2011; Wang et al., 2011). Therefore, we cannot rule out the possibility that GsMTx4 may also directly protect oligodendrocytes through several mechanisms that are independent of Piezo1. More recently, Wang et al. (2019) have shown that Yoda-1 
induces $\mathrm{Ca}^{2+}$ influx and calpain activation in the PC12 neuronal cell line and enhances oxygen/glucose deprivation-induced apoptosis. This may help to explain the data presented here, which show that Yoda-1 triggers axonal damage and demyelination and that GsMTx4 peptide attenuates both psychosine and LPC-induced demyelination. Taken together, our data suggest that blocking $\mathrm{Ca}^{2+}$ influx through mechanosensitive channels in neurons and oligodendrocytes exposed to toxins, such as psychosine and LPC, attenuates neurodegeneration and demyelination. Moreover, GsMTx4 may be a useful adjunct therapeutic for the treatment of neuroinflammatory disorders of the CNS. However, other $\mathrm{Ca}^{2+}$ independent processes could also be involved and, therefore, rigorous testing of this hypothesis is still required.

\section{Piezo1 channels and the role of mechanical cues in CNS myelination}

Piezo1 proteins (>2,500 amino acids) (Coste et al., 2010; Coste et al., 2012) trimerize to form non-selective cation channels (Gnanasambandam et al., 2015) with extracellular mechanosensing 'propeller blades' that facilitate the mechanogating mechanism of the ion channel (Zhao, Zhou, Li, \& Xiao, 2018). Traction forcemediated activation of Piezol channels in neural stem cells causes $\mathrm{Ca}^{2+}$ influx, resulting in nuclear localisation of the mechanoresponsive transcriptional coactivators yes-associated protein (Yap) and transcriptional coactivator with PDZ-binding motif (Taz) (Pathak et al., 2014). Yap/Taz coactivators also regulate myelin formation in both Schwann cells (Fernando et al., 2016; Grove et al., 2017) and oligodendrocytes (Shimizu et al., 2017). The function of Yap/Taz is to integrate biochemical and mechanical signals within cells, drive the Hippo signalling pathway, and control expression of $\alpha 6$ integrin subunits. Therefore, oligodendrocytes are mechanosensitive and recent studies have shown that oligodendrocyte precursors (OPs) grown on soft substrates tend to remain as undifferentiated progenitor cells, whereas stiffer substrates promote differentiation of OPs into myelin basic protein (MBP)-expressing mature oligodendrocytes (Jagielska et al., 2017; Jagielska et al., 2012; Lourenco \& Graos, 2016; Lourenco et al., 2016). As shown here, GsMTx4 is an important pharmacological tool that will enable further investigation of the importance of mechanical guidance cues in regulating CNS myelin formation. Indeed, in recent years the process of myelin formation has caught the attention of mechanobiologists interested in understanding how oligodendrocytes determine which axons to myelinate and if mechanical cues, such as axonal curvature, are important for that 'decision 
process' (Almeida, 2018; Bechler et al., 2018). As OPs begin to differentiate, they extend membrane processes that detect and bind axonal laminin-2 through $\alpha 6 \beta 1$ integrin adhesion molecules present on their surface. Binding of laminin-2 and $\alpha 6 \beta 1$ integrins activates the focal adhesion kinases (FAKs), Fyn and talin, which trigger mechanotransduction pathways that lead to increased MBP expression and oligodendrocyte cell maturation (Colognato, Ramachandrappa, Olsen, \& ffrenchConstant, 2004). However, much less is known about the mechanotransduction pathways that are activated specifically in neurons during myelin formation. During the initiation phase of myelination, as the oligodendrocyte begins to wrap around the axon, it must generate forces that will be transferred to, and detected by, the neuron. Given its role as a mechanosensitive channel, Piezol may be one of several candidate proteins involved in detecting traction forces generated by myelinating oligodendrocytes as they wrap around the axon's surface. Traction force-mediated activation of Piezo1 channels in neural stem cells has been shown to cause an influx of $\mathrm{Ca}^{2+}$ and results in nuclear localisation of Yap/Taz transcriptional coactivators (Pathak et al., 2014). Moreover, our data suggest that Piezo1 is a negative regulator of myelination. Recent reports suggest that shear stress-induced opening of endothelial Piezol channels activates the $\mathrm{Ca}^{2+}$-dependent protease calpain (Li et al., 2014) which, in turn, can cleave talin (a protein that links membrane integrins and the actin cytoskeleton), thus modifying integrin-mediated cell adhesion (McHugh et al., 2010). Piezo1 activation also recruits the small GTPase, R-Ras, to endoplasmic reticulum leading to calcium release from internal stores. Therefore, overactivation of Piezo1, via Yoda-1, may cause demyelination of CNS axons through excessive calpainmediated destabilisation of integrin signalling. It will be important to test this hypothesis in future studies. We also show here that blocking Piezo1 channels, using GsMTx4, promotes myelination in organotypic slice cultures under control conditions, strengthening the argument that Piezo1 is a negative regulator of CNS myelination. By blocking Piezo1 channels, GsMTx4 likely blocks excessive influx of $\mathrm{Ca}^{2+}$ into neuronal axons which may inhibit calpain-mediated destabilisation of integrin attachments and promote myelin formation. Once again, we emphasise that further work is needed to test these hypotheses and connect the missing links between Piezo1 activation and demyelination/ neuronal damage; as illustrated in schematic Fig. 12. 


\section{Conclusion}

Our data suggest that the mechanosensitive channel blocking peptide, GsMTx4, has neuroprotective properties, prevents LPC-induced astrocyte cell death, inhibits CNS demyelination caused by both LPC and psychosine, and promotes developmental myelin formation in the absence of any CNS insults. Moreover, results from this study reveal Piezol as a potential new drug target for demyelinating diseases and provide a mechanistic rationale for further development of mechanosensitive channel blockers that could enhance myelin repair in the damaged central nervous system. 

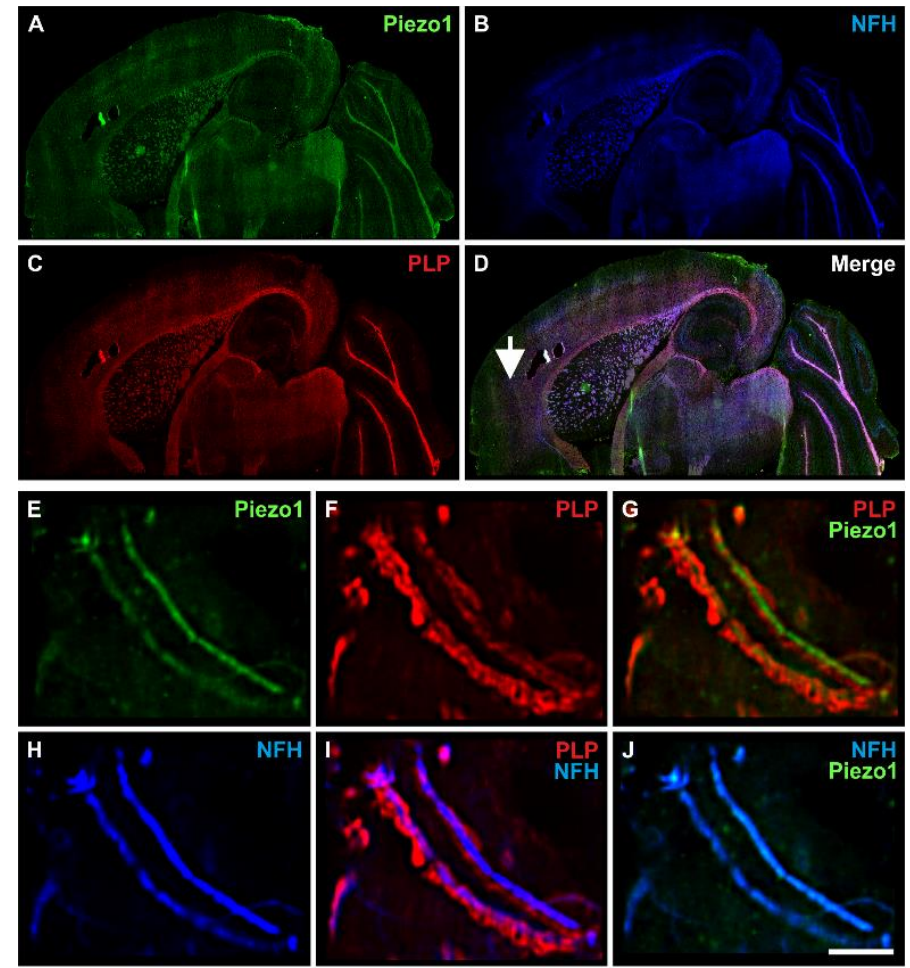

Figure 1: Piezo1 localises to neurofilament-positive axons in the mouse brain. The brains of postnatal day 36 (5-week old) mice were cryosectioned in the horizontal plane (-4.5 mm with respect to Bregma) and immunofluorescently labelled for (A) Piezo1 (green), (B) neurofilament H (NFH; blue), (C) proteolipoprotein (PLP; red) and (D) the merged image of all three channels. To determine if Piezo1 co-localises with neurons or oligodendrocytes, images of the frontal cortex (white arrow) were captured at high magnification. The frontal cortex is a region of the brain with sparsely myelinated axons running relatively parallel to one another. From qualitative analysis of these images, it was evident that Piezo1 (E) does not colocalise with PLP-labelled myelin (F, G), but instead with myelinated neuronal axons $(\mathbf{H}, \mathbf{I}, \mathbf{J})$ Scale bar $=15 \mu \mathrm{m}$. 

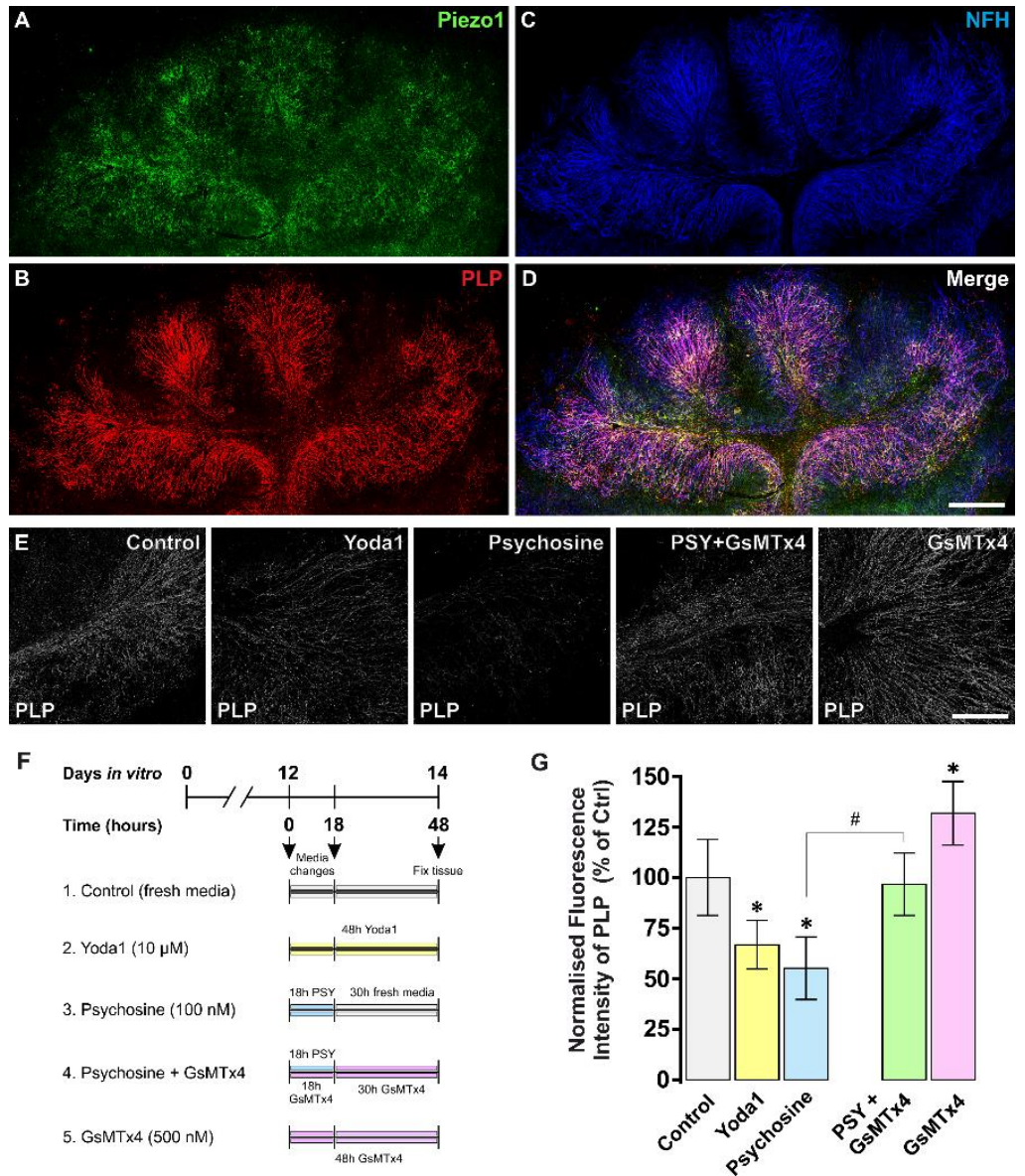

Figure 2: Piezo1 regulates CNS myelination. To examine if Piezo1 activation regulates CNS myelination, organotypic cerebellar slices were cultured for 14 days in vitro (DIV) and then fixed and immunofluorescently-labelled for (A) Piezo1 (green), (B) PLP (red), (C) NFH (blue), and (D) the merged image of all three channels. (E) Exposure of cerebellar slices to $10 \mu \mathrm{M}$ Yoda-1 for $48 \mathrm{hr}$ induced demyelination as assessed by PLP fluorescence intensity. On the contrary, treatment of slices with $500 \mathrm{nM}$ GsMTx4 promoted PLP expression in slice cultures. As previously described (Misslin et al., 2017), $18 \mathrm{hr}$ exposure to $100 \mathrm{nM}$ psychosine (PSY) induced demyelination. However, co-treatment of slices with PSY + GsMTx4 prevented demyelination. (F) Schematic diagram explaining the experimental protocol for toxin-induced demyelination of ex vivo brain slice cultures. Cerebellar slices were cultured for 12 DIV and then treated for $18 \mathrm{hr}$ with $100 \mathrm{nM}$ PSY to trigger demyelination. After $18 \mathrm{hr}$, slices were transferred to fresh medium and allowed to demyelinate for a further $30 \mathrm{hr}$. Slices were also treated with either $500 \mathrm{nM}$ GsMTx 4 or $10 \mu \mathrm{M}$ Yoda-1 to assess the effects of blocking or activating Piezo1 channels on myelination, respectively. (G) Immunofluorescence data are presented as mean \pm SEM $(n=5)$. Repeated measures one-way ANOVAs with Holm-Sidak post-hoc tests were performed. * represents a statistically significant difference $(p<0.05)$ from control and \# represents a statistically significant difference $(\mathrm{p}<0.05)$ between psychosine and PSY + GsMTx4 groups. 


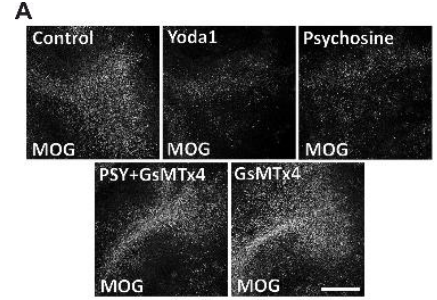

C

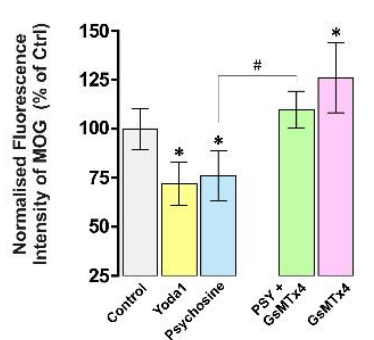

E

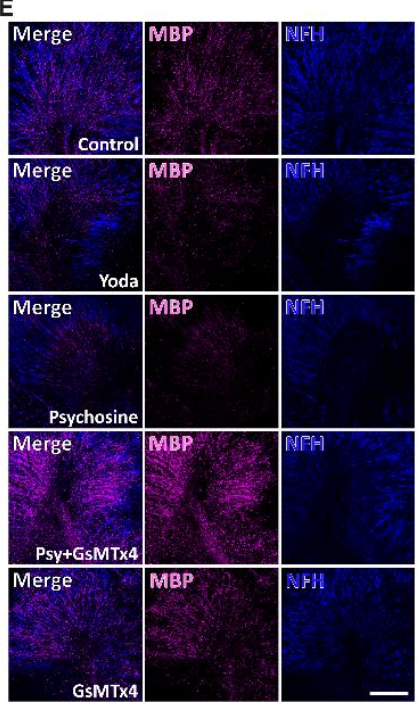

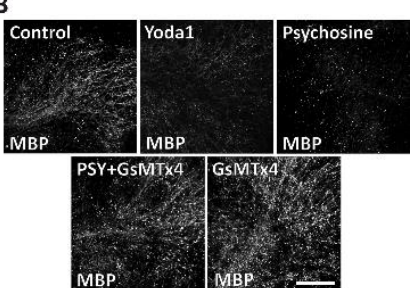

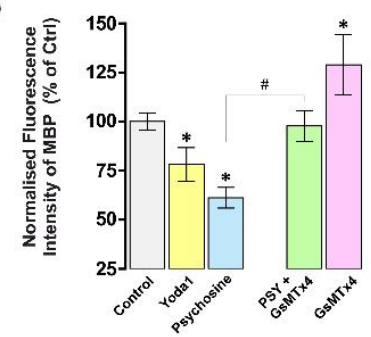

F
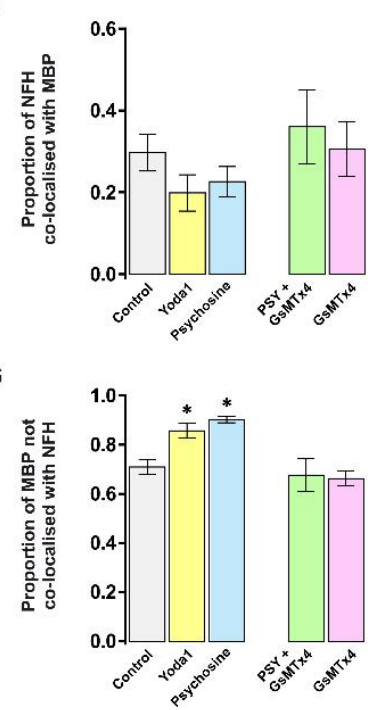

Figure 3: GsMTx4

prevents psychosine-

induced demyelination of organotypic cerebellar

slice cultures. The demyelination protocol described in Fig. 2F was repeated and the levels of (A) myelin oligodendrocyte glycoprotein (MOG) and (B) myelin basic protein (MBP) were quantified by immunofluorescence. Scale bar $=150 \mu \mathrm{m}$. The results for (C) MOG and (D) MBP closely matched those for PLP expression (see Fig. $2 \mathrm{G})$, i.e. Yoda-1 induced demyelination of cerebellar slice cultures, whereas GsMTx4 enhanced myelination and prevented PSY-induced demyelination. (E) To

assess if this decrease in the fluorescence intensity of myelin-associated proteins is accompanied by a stripping of the myelin sheath from axons, the proportion of NFH staining co-localised with MBP was quantified. (F) There were no statistically significant differences between treatment groups. However, both Yoda1 $(10 \mu \mathrm{M})$ and PSY $(100 \mathrm{nM})$ caused an increase in myelin debris compared to control slices. (G) Myelin debris was quantified as MBP staining which was not co-localised with an NFH-positive axon. GsMTx4 (500 nM) prevented PSY-induced myelin debris accumulation. Data are presented as mean $\pm \operatorname{SEM}(n=5)$. Repeated measures one-way ANOVAs with Holm-Sidak post-hoc tests were performed. * represents a statistically significant difference $(\mathrm{p}<0.05)$ from control and \# represents a statistically significant difference $(\mathrm{p}<0.05)$ between psychosine and PSY + GsMTx4 groups. 
A

B
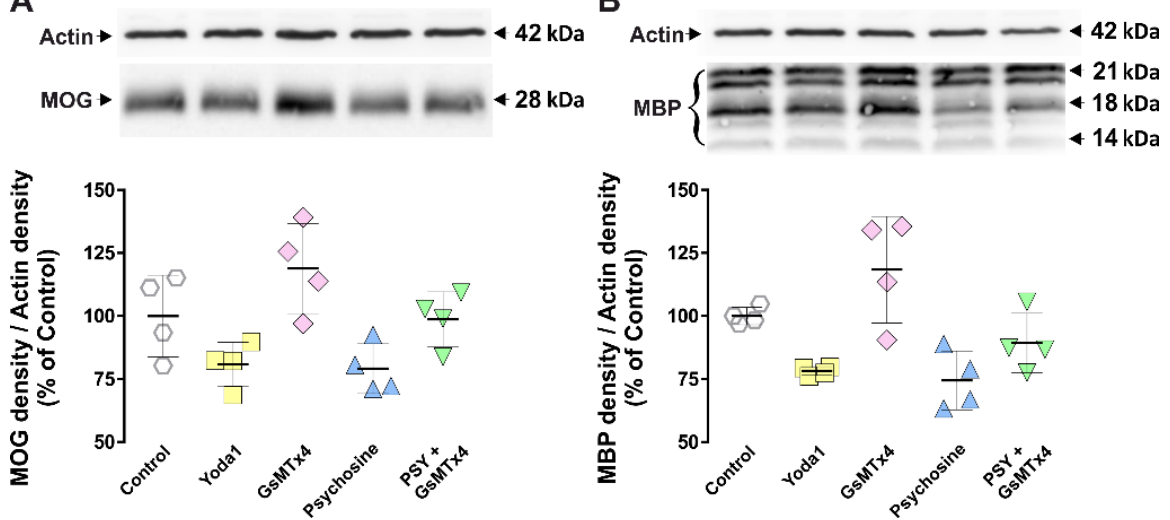

Figure 4: Western blot confirming changes in myelin-associated proteins in response to Yoda1 and GsMTx4 treatments. The demyelination protocol (described in Fig. 2F) was repeated in cerebellar slice cultures and protein samples were prepared. Changes in the levels of (A) myelin oligodendrocyte glycoprotein (MOG) and (B) myelin basic protein (MBP) were quantified using Western blotting. There were no statistically significant differences in MOG or MBP protein levels between treatment groups (repeated measures one-way ANOVAs with Holm-Sidak post-hoc tests were performed), although quantification of the relative abundances in myelin-associated protein appears to follow the same pattern of expression to that described in Fig. 3.

A

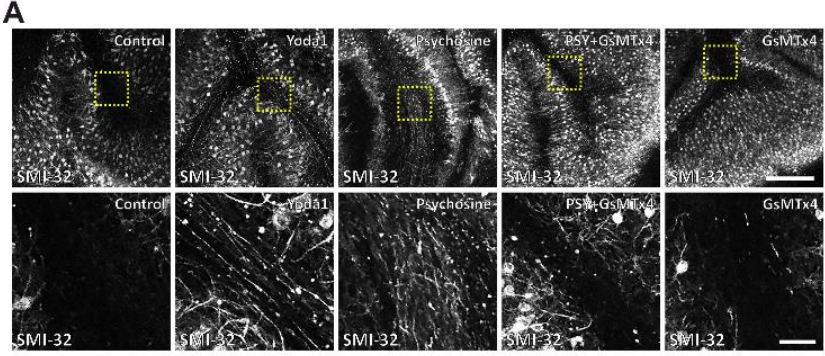

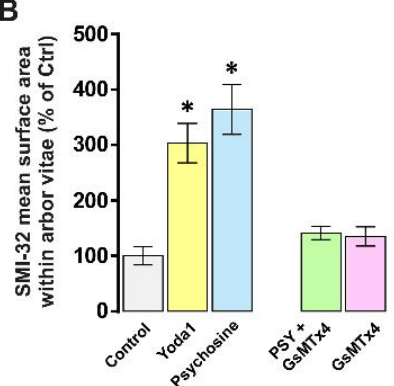

Figure 5: GsMTx4 inhibits psychosine-induced axonal injury in organotypic cerebellar slice cultures. OCS cultures were exposed to the same experimental protocol described in Fig. 2F. (A) Slices were immunofluorescently stained for the axonal damage marker, SMI-32 (n = 5), a non-phosphorylated epitope of neurofilament $\mathrm{H}$ which is constitutively expressed in the soma of neurons but absent in healthy axons. Scale bar $=150 \mu \mathrm{m}$. Changes in axonal SMI-32 expression were quantified by calculating the mean surface area stained within the cerebellar arbor vitae axons (yellow boxes; scale bar $=30 \mu \mathrm{m})$. (B) Yoda-1 $(10 \mu \mathrm{M})$ and PSY $(100 \mathrm{nM})$ caused an increase in axonal damage. Co-treatment of slices with GsMTx4 (500 nM) prevented PSY-induced axonal injury. Immunofluorescence data are presented as mean \pm SEM $(n=5)$. Repeated measures one-way ANOVAs with Holm-Sidak post-hoc tests were performed. * represents a statistically significant difference $(\mathrm{p}<0.05)$ from control. 

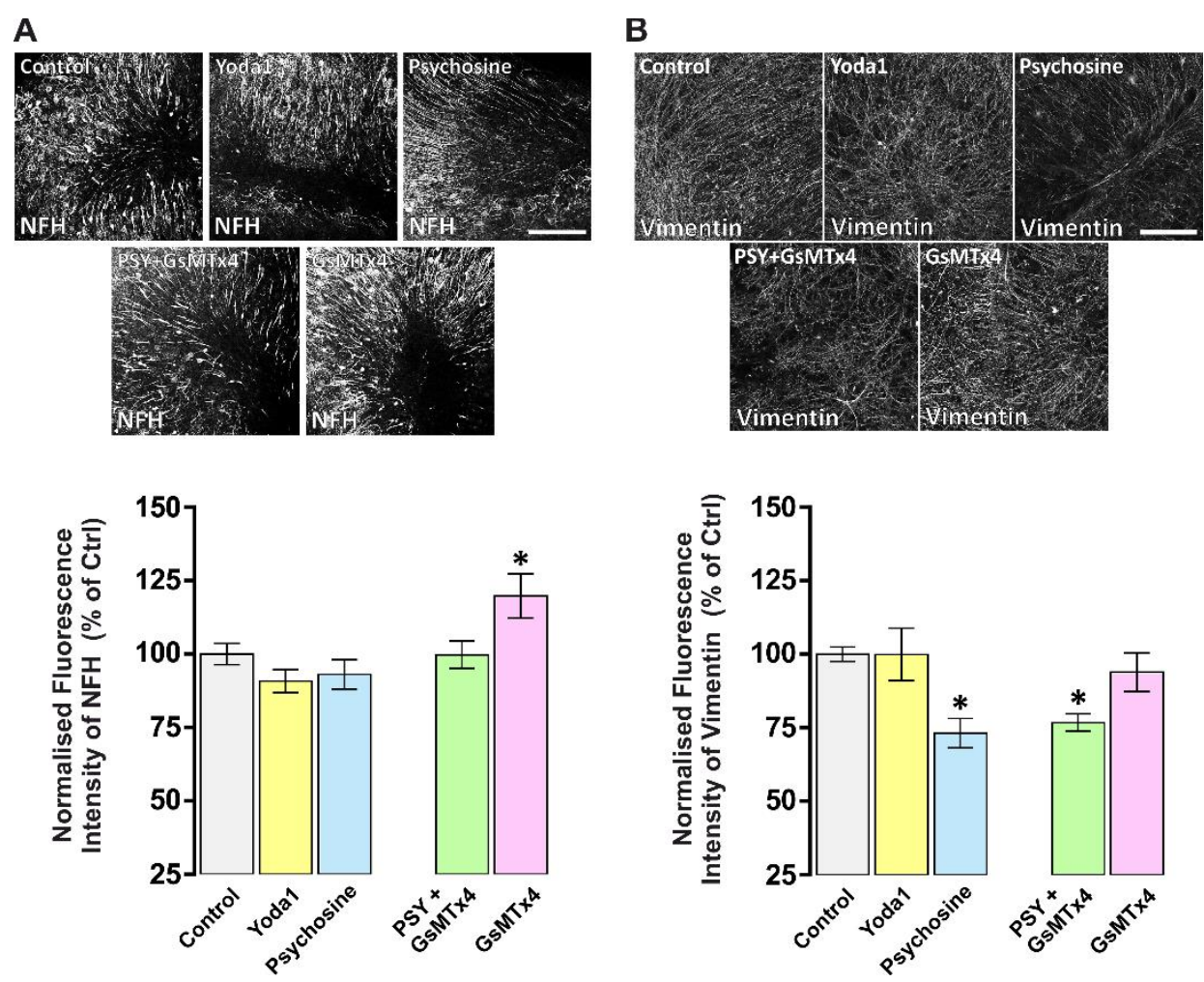

Figure 6: GsMTx4 does not prevent psychosine-induced decreases in neural precursor cells. OCS cultures were exposed to the same experimental protocol described in Fig. 2F. (A) Slices were then immunofluorescently-stained for phosphorylated neurofilament $\mathrm{H}(\mathrm{NFH})$, a marker of axonal integrity, and changes in NFH fluorescence intensity were quantified $(\mathrm{n}=11)$. Scale bar $=150 \mu \mathrm{m}$. Neither Yoda-1 nor PSY caused any significant changes in NFH expression. GsMTx4, on the other hand, caused an increase in NFH expression suggestive of a neuroprotective effect in OCS cultures. (B) Next, the responses of neural precursor cells (NPC) to Yoda-1, PSY and GsMTx4 exposure were quantified by assessing changes in Vimentin immunofluorescence $(n=6)$. Scale bar $=150 \mu \mathrm{m}$. PSY caused a significant decrease in Vimentin fluorescence intensity. However, co-treatment of slices with PSY + GsMTx4 did not rescue NPC degeneration. Immunofluorescence data are presented as mean $\pm \mathrm{SEM}$. Repeated measures one-way ANOVAs with Holm-Sidak post-hoc tests were performed. * represents a statistically significant difference $(p<0.05)$ from the control group. 

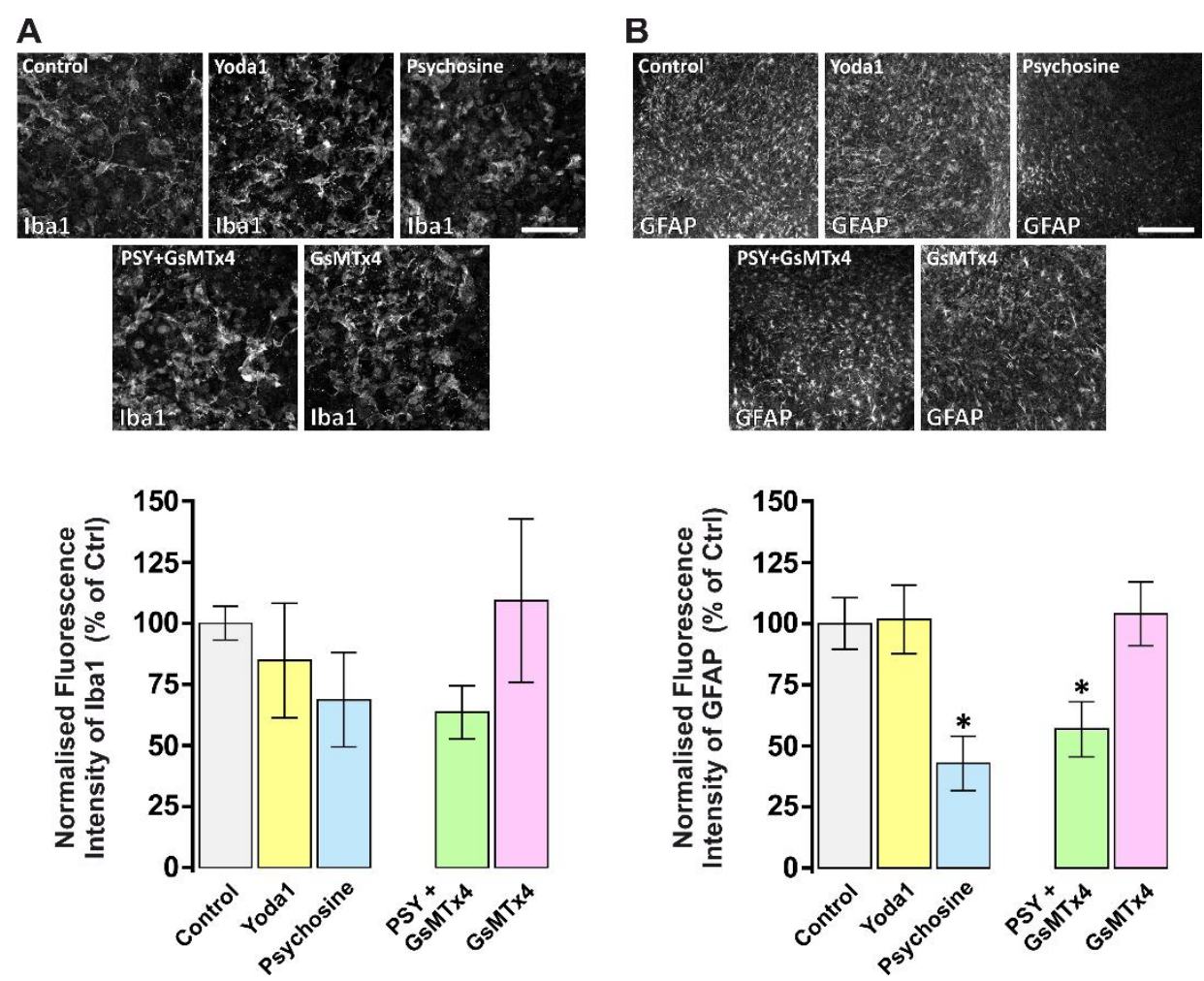

Figure 7: GsMTx4 does not attenuate psychosine-induced astrocyte damage in vitro. OCS cultures underwent the same experimental protocol described in Fig. 2F. (A) Slices were then immunofluorescently-stained for ionized calcium binding adaptor molecule $1(I b a 1 ; n=6)$ to assess microglial reactivity in response to Yoda-1, PSY and GsMTx4. Scale bar $=150 \mu \mathrm{m}$. Neither psychosine nor Yoda1 caused any significant change in microglial responses, assessed as a change in Iba1 fluorescence intensity. Similarly, treating slices with GsMTx4 (500 nM for $48 \mathrm{hr}$ ) had no significant effect on microglial reactivity in OCS cultures. (B) Next, OCS cultures were stained for glial fibrillary acidic protein (GFAP) to study the effects of Yoda-1, PSY and GsMTx4 on astrocytes. Psychosine decreased GFAP fluorescence intensity in OSC cultures suggesting a toxic effect on astrocytes. Yoda1 and GsMTx4 had no effect on GFAP levels in OCS cultures. Moreover, GsMTx4 did not rescue psychosine-induced astrocyte damage. Immunofluorescence data are presented as mean \pm SEM. Repeated measures one-way ANOVAs with Holm-Sidak post-hoc tests were performed. * represents a statistically significant difference $(p<0.05)$ from the control group. 
A

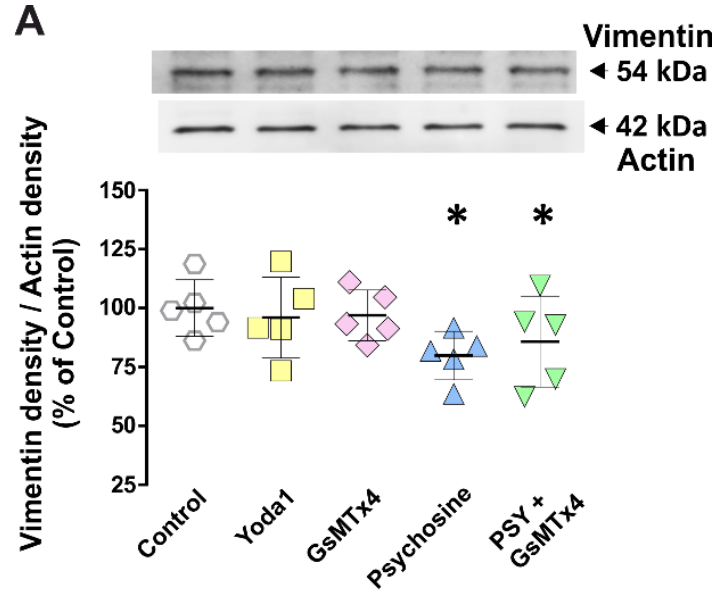

B

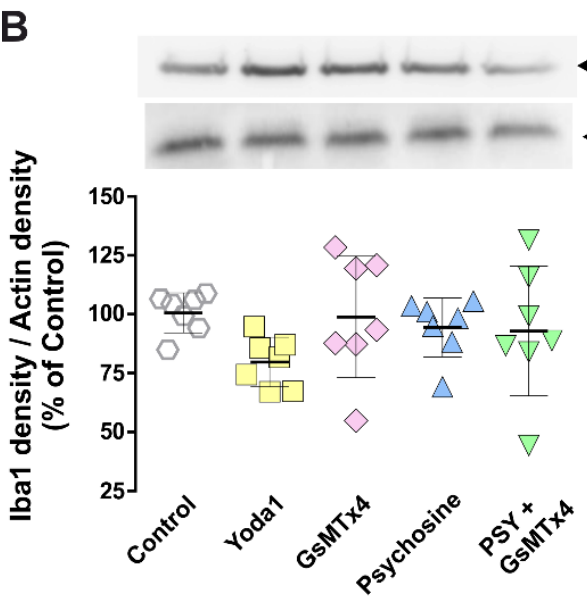

C

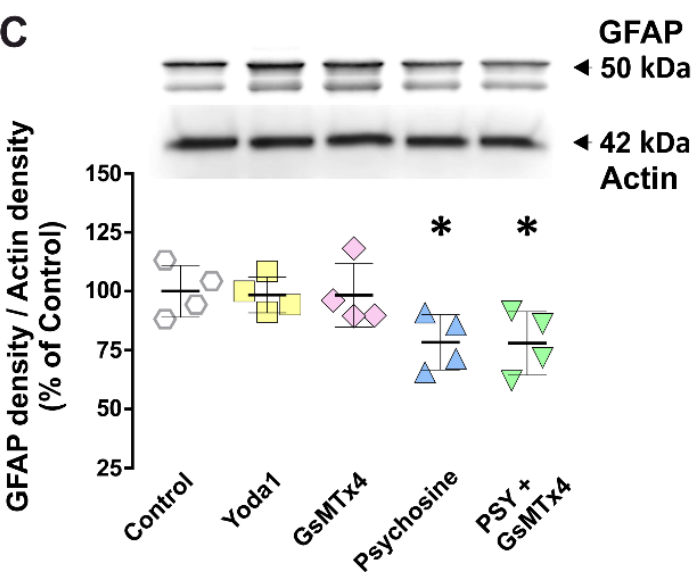

Figure 8: Western blot confirming that GsMTx4 does not rescue neural precursor cell degeneration or astrocyte toxicity induced by psychosine. The treatment protocol (described in Fig. 2F) was repeated in cerebellar slice cultures and protein samples were prepared. Changes in the levels of (A) Vimentin, (B) Iba1, and (C) GFAP were quantified using Western blotting. There was a statistically significant decrease in (A) Vimentin protein levels following psychosine exposure. However, GsMTx4 did not protect NPCs from cytotoxicity. Yoda1, GsMTx4 and psychosine had no effects on Iba1 protein abundance, suggesting microglial reactivity was unchanged following each pharmacological treatment. (C) Psychosine also caused a significant decrease in GFAP protein abundance relative to control slices, but this damage to astrocytes was not rescued by cotreatment with GsMTx4. These results matched that found in Fig. 7B using immunofluorescence techniques. Repeated measures one-way ANOVAs with Holm-Sidak post-hoc tests were performed. * represents a statistically significant difference $(\mathrm{p}<0.05)$ from control. 


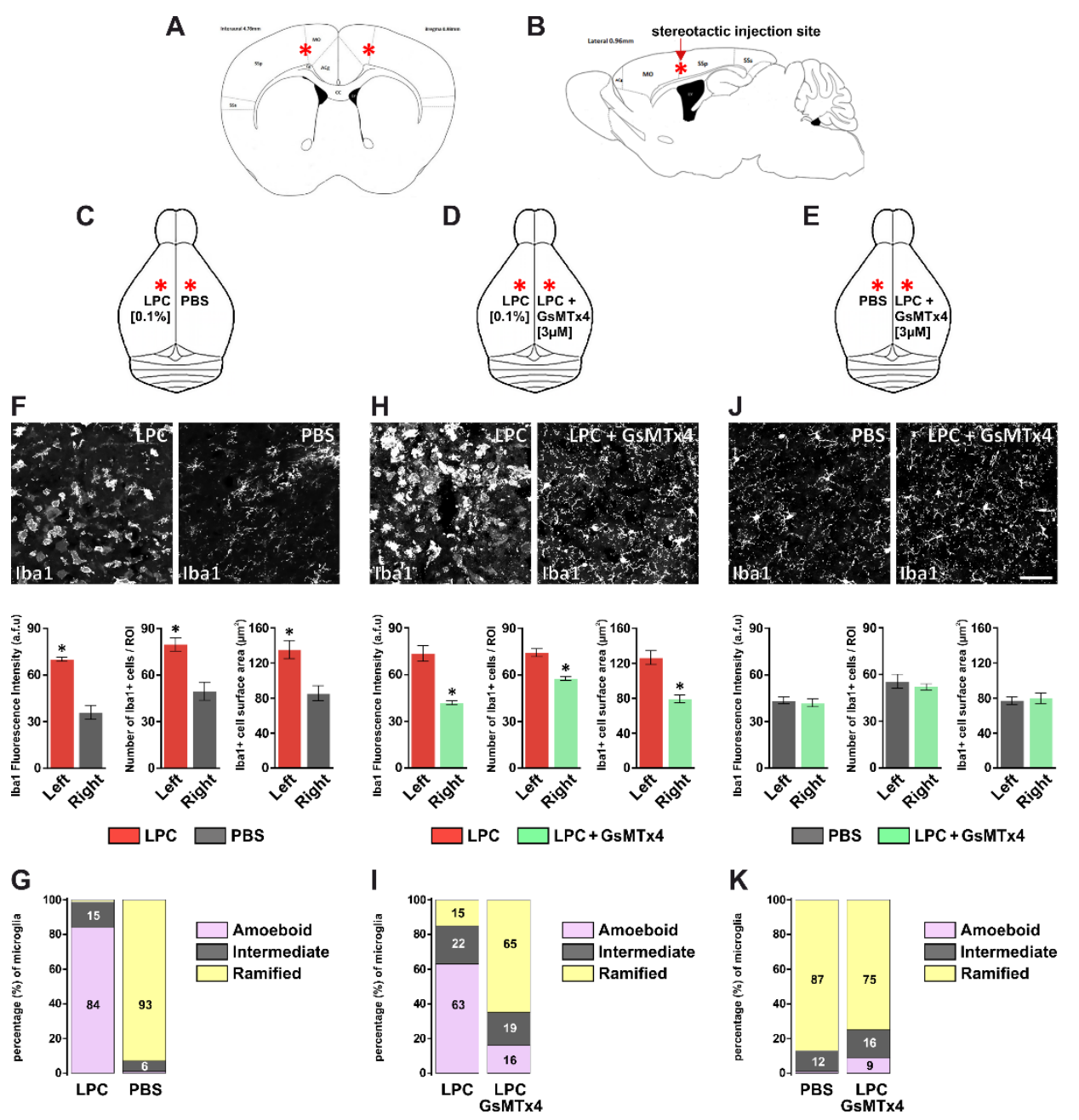

Figure 9: GsMTx4 attenuates microglial reactivity induced by lysophosphatidylcholine in vivo. (A, B) Adult C57BL/6 mice were stereotactically-injected in the left and right cerebral hemispheres with either: (C) LPC 0.1\% (left) vs PBS (right) ( $\mathrm{n}=2$ ); (D) LPC $0.1 \%$ (left) vs LPC $0.1 \%+3 \mu \mathrm{M}$ GsMTx4 (right) $(\mathrm{n}=4)$; or (E) PBS (left) vs LPC $0.1 \%+3 \mu \mathrm{M}$ GsMTx4 (right) $(n=4)$. Mice were sacrificed 4 days post-surgery and perfused with $4 \%$ PFA and the brains were sectioned for immunofluorescence. (F) LPC caused significant microglial reactivity, measured as an increase in Iba1 fluorescence intensity, an increase in the number of Iba1 + cells in the lesion core, and an increase in the surface area of individual microglial cells which is caused by (G) a change from ramified (PBS) to amoeboid-like cell morphology in the LPC-treated left hemisphere. (H) Co-injection of LPC + GsMTx4 in the right cerebral cortex prevented the increase in microglial reactivity measured in the LPC-treated left hemisphere. (I) Comparison of Iba $1+$ microglia in the LPC-exposed left hemisphere (where $\sim 63 \%$ of cells displayed an amoeboid-like morphology) with the LPC + GsMTx 4 treated right hemisphere (where only $\sim 16 \%$ of cells appeared amoeboid-like) reveals that GsMTx4 inhibits 
morphological changes in microglial cells which remain in the ramified non-reactive state. (J) As such, there were no significant differences in Ibal fluorescence intensity, Iba $1+$ cell numbers or Iba1+ cell surface area between the PBS-treated left hemisphere and LPC + GsMTx4 treated right hemisphere. Scale bar $=100 \mu \mathrm{m}$. (K) Moreover, a similar percentage of microglial cells showed quiescent ramified morphologies in PBS (87\%) versus LPC + GsMTx4 (75\%) exposed cerebral hemispheres. Immunofluorescence data are presented as mean \pm SEM. Two-way ANOVAs with Holm-Sidak post-hoc tests were performed to compare the left and right hemispheres of each treatment group. * represents a statistically significant difference $(\mathrm{p}<$ 0.05 ) between the left and right hemisphere within each group. The analysis of proportions was carried out using chi-squared $\left(\chi^{2}\right)$ tests in conjunction with the Bonferroni correction for multiple comparisons. 
A
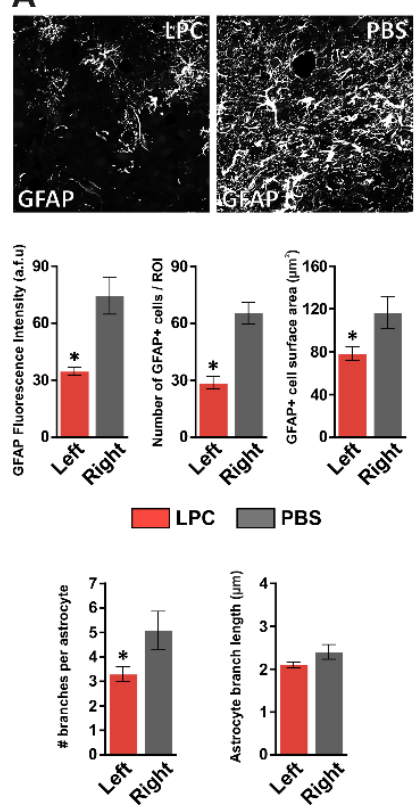

B
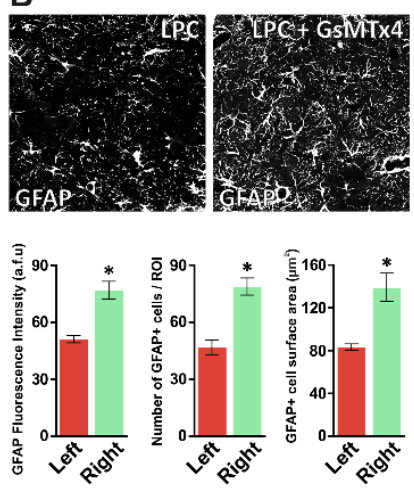

$\square$ LPC $\square$ LPC+GsMTx4

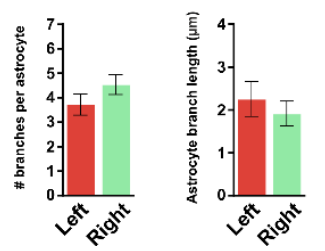

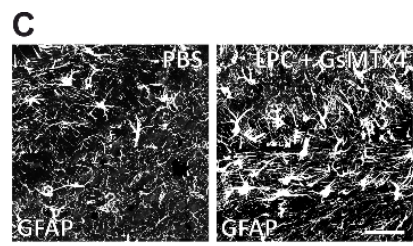

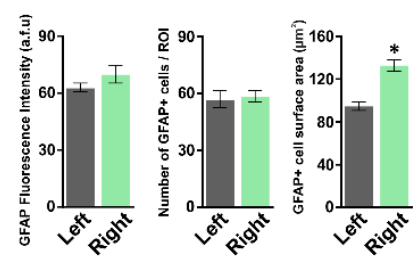

$\square$ PBS $\square$ LPC+GsMTx4

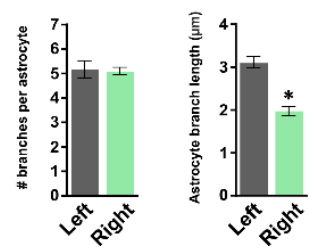

Figure 10: GsMTx4 attenuates astrocyte cell death induced by lysophosphatidylcholine in vivo. (A) LPC caused a significant reduction in GFAP fluorescence intensity and reduced the number of GFAP+ astrocytes at the site of injury. The mean surface area of cortical astrocytes was also reduced suggesting that LPC exposure caused astrocyte cell death within the lesion core. This was also reflected in a reduction in the number of branches per astrocyte. (B) Coinjection of LPC + GsMTx4, however, prevented astrocyte toxicity. (C) GFAP fluorescence intensity and the number of GFAP+ astrocytes at the site of injection were similar in PBS vehicle control left hemispheres and LPC + GsMTx4 right hemispheres. However, the mean surface area of GFAP+ astrocytes increased in the LPC + GsMTx4 hemisphere, whilst the average length of each branch was reduced, suggesting that GsMTx4 not only protects astrocytes from LPC-induced cell death, but also facilitates subsequent hypertrophy and reactivity, possibly expediting recovery from CNS injury. Scale bar $=100 \mu \mathrm{m}$. Immunofluorescence data are presented as mean \pm SEM. Two-way ANOVAs with Holm-Sidak post-hoc tests were performed to compare the left and right hemispheres of each treatment group. * represents a statistically significant difference $(\mathrm{p}<0.05)$ between the left and right hemisphere within each group. 
A

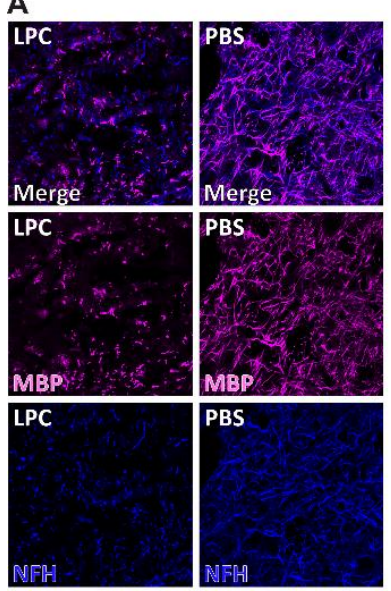

B

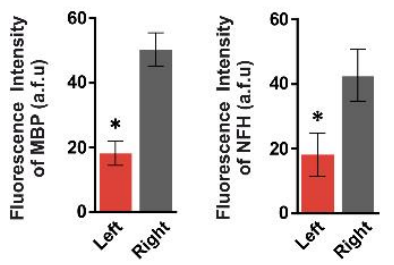

$\square$ LPC $\square$ PBS

C
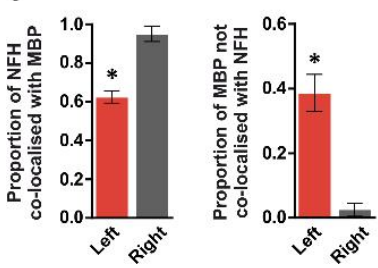

D

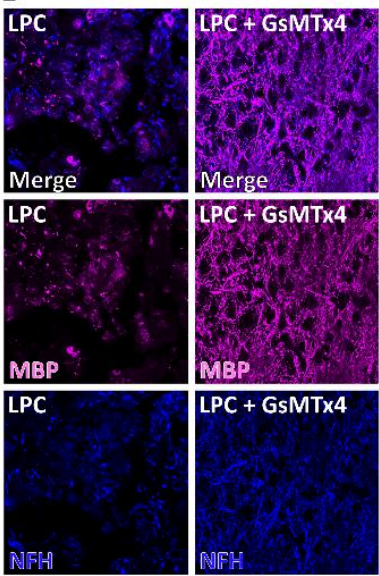

E

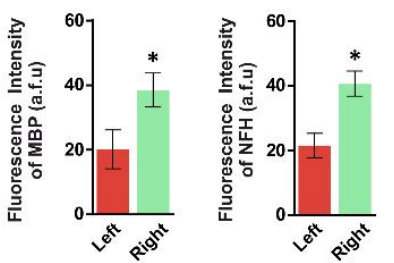

$\square$ LPC $\square$ LPC + GsMTx4
F

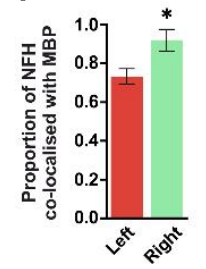

G
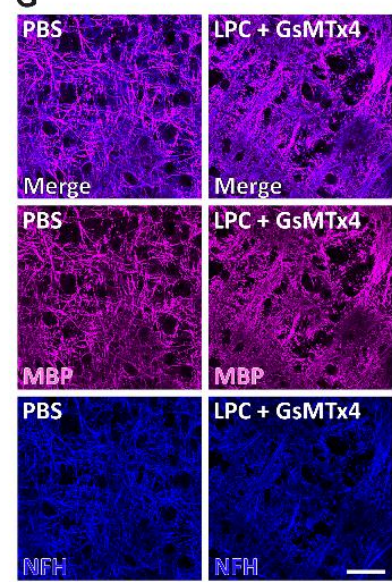

H

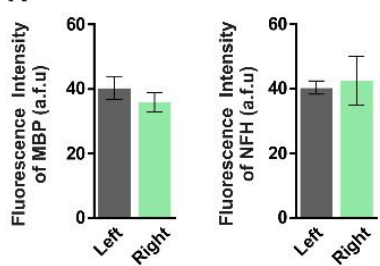

$\square$ PBS $\square$ LPC + GsMTx4

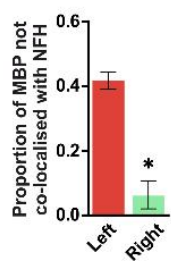

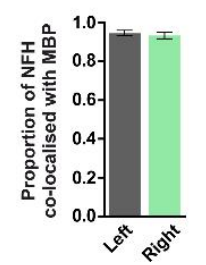

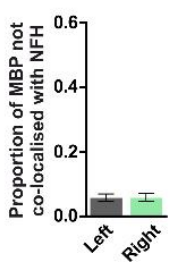

Figure 11: GsMTx4 is neuroprotective and prevents LPC-induced demyelination of cortical axons in vivo. Adult C57BL/6 mice were randomly assigned into one of three groups, as described in Fig. 9C $-\mathrm{E}$, and injected with either PBS, LPC $0.1 \%$ or LPC + GsMTx4 $(3 \mu \mathrm{M})$ into the left and right cerebral hemispheres. (A) LPC caused significant demyelination and axonal damage, (B) measured as a decrease in myelin basic protein (MBP) and neurofilament $\mathrm{H}$ (NFH) immunofluorescence, respectively. (C) Moreover, LPC caused a decrease in the proportion of NFH-labelled axons co-localised with MBP staining, suggesting that the myelin sheath was stripped from axons following LPC exposure. As such, LPC caused an increase in the accumulation of myelin debris in the lesion core, measured as an increase in MBP-staining which was not co-localised with NFH. (D, E, F) Co-injection of GsMTx4 prevented LPCinduced axonal injury and demyelination. The neuroprotective and myelin-preserving properties of GsMTx4 were confirmed by comparing (G) PBS vehicle control injected hemispheres (left) with LPC + GsMTx4 hemispheres (right). Scale bar $=100 \mu \mathrm{m}$. (H, I) Cortical axons exposed to LPC + GsMTx4 displayed similar levels of MBP and NFH expression to PBS-treated hemispheres. Moreover, there was no reduction in NFH+ axons which were co-localised with MBP and no accumulation of myelin debris when GsMTx4 was 
co-injected with LPC. This confirmed the myelin-preserving properties of blocking Piezo1 channels. Immunofluorescence data are presented as mean \pm SEM. Two-way ANOVAs with Holm-Sidak post-hoc tests were performed to compare the left and right hemispheres of each treatment group. $*$ represents a statistically significant difference $(\mathrm{p}<0.05)$ between the left and right hemisphere within each group.

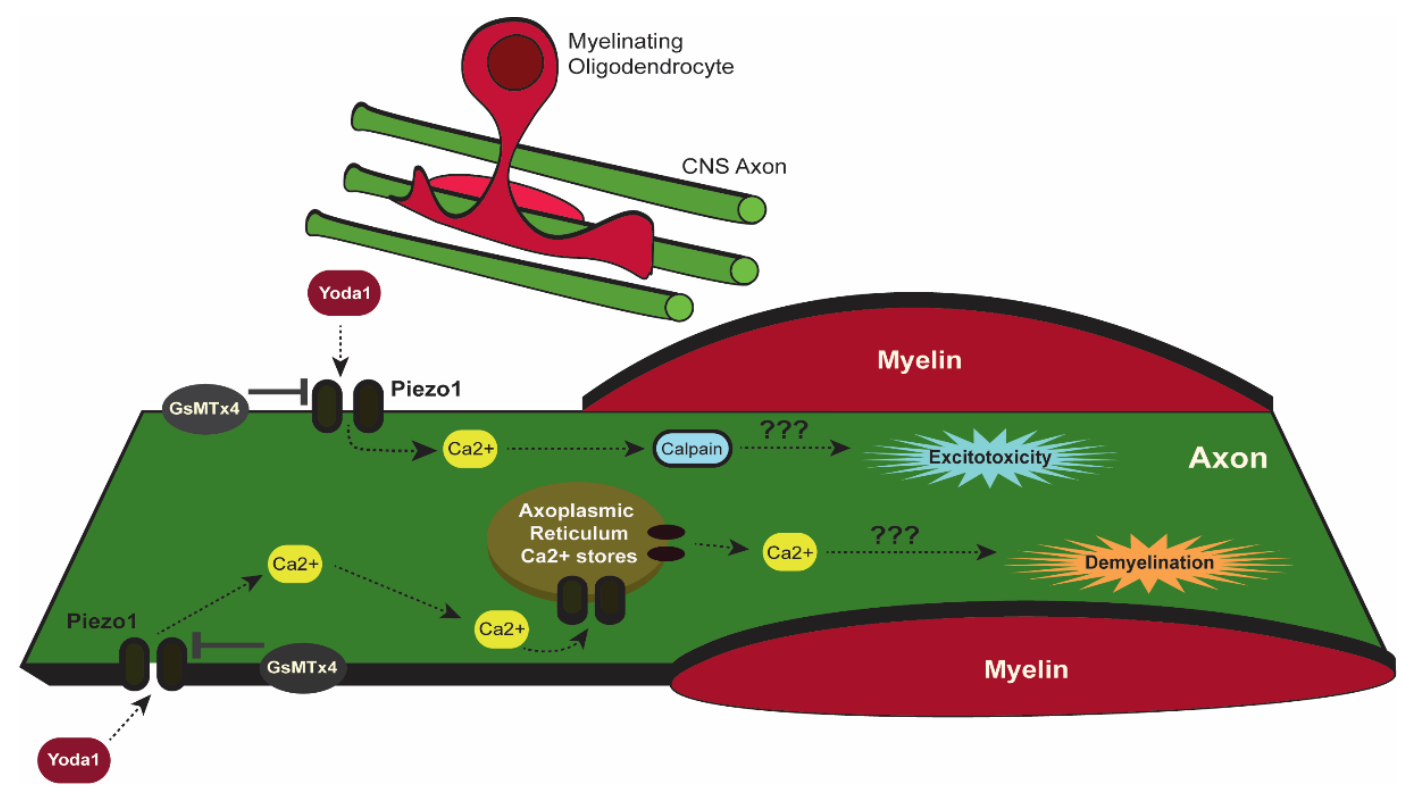

Figure 12: Proposed mechanism to explain Yoda1-mediated demyelination of CNS axons and how GsMTx4 may prevent the neuro-damaging actions of Piezo1 overactivation. Yoda1 can activate Piezo1 channels present on CNS axons. This may promote the influx of extracellular $\mathrm{Ca}^{2+}$ into the neuron which, in turn, could trigger calcium-induced calcium release (CICR) from intracellular stores. Yoda1 has also been shown to activate calpain signalling in neurons leading to neurodegeneration (Wang et al. 2019). This may explain how Yoda1 causes demyelination of CNS axons, i.e. partly mediated through excitotoxicity and neuronal damage. GsMTx4 acts to block mechanosensitive $\mathrm{Ca}^{2+}$ channels such as Piezo1. By inhibiting excessive $\mathrm{Ca}^{2+}$ influx or CICR from internal stores, GsMTx4 may prove a useful experimental tool to probe further the role of Piezo1 in myelination of the central nervous system. 


\section{References}

Allen, D. G., Whitehead, N. P., \& Froehner, S. C. (2016). Absence of Dystrophin Disrupts Skeletal Muscle Signaling: Roles of $\mathrm{Ca}^{2+}$, Reactive Oxygen Species, and Nitric Oxide in the Development of Muscular Dystrophy. Physiol Rev, 96(1), 253-305. doi:10.1152/physrev.00007.2015

Almeida RG (2018) The Rules of Attraction in Central Nervous System Myelination. Front Cell Neurosci. 12: 367. doi: 10.3389/fncel.2018.00367

Azuma, M., \& Shearer, T. R. (2008). The role of calcium-activated protease calpain in experimental retinal pathology. Surv Ophthalmol, 53(2), 150-163. doi:10.1016/j.survophthal.2007.12.006

Baloh, R. H. (2008). Mitochondrial dynamics and peripheral neuropathy. Neuroscientist, 14(1), 12-18. doi:10.1177/1073858407307354

Barsukova, A. G., Forte, M., \& Bourdette, D. (2012). Focal increases of axoplasmic Ca2+, aggregation of sodium-calcium exchanger, $\mathrm{N}$-type $\mathrm{Ca} 2+$ channel, and actin define the sites of spheroids in axons undergoing oxidative stress. J Neurosci, 32(35), 1202812037. doi:10.1523/jneurosci.0408-12.2012

Bechler ME, Swire M, ffrench-Constant C. (2018) Intrinsic and adaptive myelination-A sequential mechanism for smart wiring in the brain. Dev Neurobiol. 78(2), 68-79. doi:10.1002/dneu.22518.

Berret E, Barron T, Xu J, Debner E, Kim EJ, Kim JH. (2017) Oligodendroglial excitability mediated by glutamatergic inputs and Nav1.2 activation. Nat Commun. 8(1), 557. doi: 10.1038/s41467-017-00688-0.

Blumenthal, N. R., Hermanson, O., Heimrich, B., \& Shastri, V. P. (2014). Stochastic nanoroughness modulates neuron-astrocyte interactions and function via mechanosensing cation channels. Proc Natl Acad Sci U S A, 111(45), 16124-16129. doi:10.1073/pnas.1412740111

Castelvetri, L. C., Givogri, M. I., Zhu, H., Smith, B., Lopez-Rosas, A., Qiu, X., . . Bongarzone, E. R. (2011). Axonopathy is a compounding factor in the pathogenesis of Krabbe disease. Acta Neuropathol, 122(1), 35-48. doi:10.1007/s00401-011-0814-2

Chang, K. J., Redmond, S. A., \& Chan, J. R. (2016). Remodeling myelination: implications for mechanisms of neural plasticity. Nat Neurosci, 19(2), 190-197. doi:10.1038/nn.4200

Colognato, H., Ramachandrappa, S., Olsen, I. M., \& ffrench-Constant, C. (2004). Integrins direct Src family kinases to regulate distinct phases of oligodendrocyte development. J Cell Biol, 167(2), 365-375. doi:10.1083/jcb.200404076

Coste, B., Mathur, J., Schmidt, M., Earley, T. J., Ranade, S., Petrus, M. J., . . Patapoutian, A. (2010). Piezo1 and Piezo2 are essential components of distinct mechanically activated cation channels. Science, 330(6000), 55-60. doi:10.1126/science.1193270

Coste, B., Xiao, B., Santos, J. S., Syeda, R., Grandl, J., Spencer, K. S., . . . Patapoutian, A. (2012). Piezo proteins are pore-forming subunits of mechanically activated channels. Nature, 483(7388), 176-181. doi:10.1038/nature10812

Evans, E. L., Cuthbertson, K., Endesh, N., Rode, B., Blythe, N. M., Hyman, A. J., . . Beech, D. J. (2018). Yoda1 analogue (Dooku1) which antagonizes Yoda1-evoked activation of Piezol and aortic relaxation. $B r \quad J$ Pharmacol, 175(10), 1744-1759. doi:10.1111/bph.14188

Fernando, R. N., Cotter, L., Perrin-Tricaud, C., Berthelot, J., Bartolami, S., Pereira, J. A., . . . Tricaud, N. (2016). Optimal myelin elongation relies on YAP activation by axonal growth and inhibition by Crb3/Hippo pathway. Nat Commun, 7, 12186. doi:10.1038/ncomms12186

Franklin, R. J., ffrench-Constant, C., Edgar, J. M., \& Smith, K. J. (2012). Neuroprotection and repair in multiple sclerosis. Nat Rev Neurol, 8(11), 624-634. doi:10.1038/nrneurol.2012.200

Frati, A., Cerretani, D., Fiaschi, A. I., Frati, P., Gatto, V., La Russa, R., . . Fineschi, V. (2017). Diffuse Axonal Injury and Oxidative Stress: A Comprehensive Review. Int J Mol Sci, 18(12), 2600. doi:10.3390/ijms 18122600

Fu, Y., Wang, H., Huff, T. B., Shi, R., \& Cheng, J. X. (2007). Coherent anti-Stokes Raman 
scattering imaging of myelin degradation reveals a calcium-dependent pathway in lyso-PtdCho-induced demyelination. $J$ Neurosci Res, 85(13), 2870-2881. doi: $10.1002 /$ jnr. 21403

Galbiati, F., Givogri, M. I., Cantuti, L., Rosas, A. L., Cao, H., van Breemen, R., \& Bongarzone, E. R. (2009). Combined hematopoietic and lentiviral gene-transfer therapies in newborn Twitcher mice reveal contemporaneous neurodegeneration and demyelination in Krabbe disease. $J$ Neurosci Res, 87(8), 1748-1759. doi:10.1002/jnr.22006

Giri, S., Jatana, M., Rattan, R., Won, J. S., Singh, I., \& Singh, A. K. (2002). Galactosylsphingosine (psychosine)-induced expression of cytokine-mediated inducible nitric oxide synthases via AP-1 and C/EBP: implications for Krabbe disease. Faseb j, 16(7), 661-672. doi:10.1096/fj.01-0798com

Gnanasambandam, R., Bae, C., Gottlieb, P. A., \& Sachs, F. (2015). Ionic Selectivity and Permeation Properties of Human PIEZO1 Channels. PLoS One, 10(5), e0125503. doi:10.1371/journal.pone.0125503

Gnanasambandam, R., Ghatak, C., Yasmann, A., Nishizawa, K., Sachs, F., Ladokhin, A. S., . . . Suchyna, T. M. (2017). GsMTx4: Mechanism of Inhibiting Mechanosensitive Ion Channels. Biophys J, 112(1), 31-45. doi:10.1016/j.bpj.2016.11.013

Gorlach, A., Bertram, K., Hudecova, S., \& Krizanova, O. (2015). Calcium and ROS: A mutual interplay. Redox Biol, 6, 260-271. doi:10.1016/j.redox.2015.08.010

Gottlieb, P. A., \& Sachs, F. (2012). Piezo1: properties of a cation selective mechanical channel. Channels (Austin), 6(4), 214-219. doi:10.4161/chan.21050

Gottlieb, P. A., Suchyna, T. M., \& Sachs, F. (2007). Properties and Mechanism of the Mechanosensitive Ion Channel Inhibitor GsMTx4, a Therapeutic Peptide Derived from Tarantula Venom. Curr Top Membr, 59, 81-109. doi:10.1016/s10635823(06)59004-0

Grove, M., Kim, H., Santerre, M., Krupka, A. J., Han, S. B., Zhai, J., . . Son, Y. J. (2017). YAP/TAZ initiate and maintain Schwann cell myelination. Elife, 6, e20982. doi:10.7554/eLife. 20982

Hauser, S. L., \& Oksenberg, J. R. (2006). The neurobiology of multiple sclerosis: genes, inflammation, and neurodegeneration. Neuron, 52(1), 61-76. doi:10.1016/j.neuron.2006.09.011

Hill, K., \& Schaefer, M. (2007). TRPA1 is differentially modulated by the amphipathic molecules trinitrophenol and chlorpromazine. J Biol Chem. 282(10), 7145-7153. doi: 10.1074/jbc.M609600200

Hsieh ST, Kidd GJ, Crawford TO, Xu Z, Lin WM, Trapp BD, Cleveland DW, Griffin JW (1994). Regional modulation of neurofilament organization by myelination in normal axons. J Neurosci. 14: 6392-6401. doi: 10.1523/JNEUROSCI.14-1106392.1994

Ilkan, Z., Wright, J. R., Goodall, A. H., Gibbins, J. M., Jones, C. I., \& Mahaut-Smith, M. P. (2017). Evidence for shear-mediated $\mathrm{Ca}(2+)$ entry through mechanosensitive cation channels in human platelets and a megakaryocytic cell line. J Biol Chem, 292(22), 9204-9217. doi:10.1074/jbc.M116.766196

Irvine, K. A., \& Blakemore, W. F. (2008). Remyelination protects axons from demyelinationassociated axon degeneration. Brain, 131(Pt 6), 1464-1477. doi:10.1093/brain/awn080

Ishibashi, T., Dakin, K. A., Stevens, B., Lee, P. R., Kozlov, S. V., Stewart, C. L., \& Fields, R. D. (2006). Astrocytes promote myelination in response to electrical impulses. Neuron, 49(6), 823-832. doi:10.1016/j.neuron.2006.02.006

Jacques-Fricke, B. T., Seow, Y., Gottlieb, P. A., Sachs, F., \& Gomez, T. M. (2006). Ca2+ influx through mechanosensitive channels inhibits neurite outgrowth in opposition to other influx pathways and release from intracellular stores. J Neurosci, 26(21), 5656-5664. doi:10.1523/jneurosci.0675-06.2006

Jagielska, A., Lowe, A. L., Makhija, E., Wroblewska, L., Guck, J., Franklin, R. J. M., . . Van Vliet, K. J. (2017). Mechanical Strain Promotes Oligodendrocyte Differentiation by Global Changes of Gene Expression. Front Cell Neurosci, 11, 93. doi:10.3389/fncel.2017.00093 
Jagielska, A., Norman, A. L., Whyte, G., Vliet, K. J., Guck, J., \& Franklin, R. J. (2012). Mechanical environment modulates biological properties of oligodendrocyte progenitor cells. Stem Cells Dev, 21(16), 2905-2914. doi:10.1089/scd.2012.0189

Kocur M, Schneider R, Pulm AK, Bauer J, Kropp S, Gliem M, Ingwersen J, Goebels N, Alferink J, Prozorovski T, Aktas O, Scheu S. (2015) IFN $\beta$ secreted by microglia mediates clearance of myelin debris in CNS autoimmunity. Acta Neuropathol Commun. 3, 20. doi: 10.1186/s40478-015-0192-4.

Koser, D. E., Thompson, A. J., Foster, S. K., Dwivedy, A., Pillai, E. K., Sheridan, G. K., . . . Franze, K. (2016). Mechanosensing is critical for axon growth in the developing brain. Nat Neurosci, 19(12), 1592-1598. doi:10.1038/nn.4394

Lacroix, J. J., Botello-Smith, W. M., \& Luo, Y. (2018). Probing the gating mechanism of the mechanosensitive channel Piezo1 with the small molecule Yoda1. Nat Commun, 9(1), 2029. doi:10.1038/s41467-018-04405-3

Li, J., Hou, B., Tumova, S., Muraki, K., Bruns, A., Ludlow, M. J., . . Beech, D. J. (2014). Piezo1 integration of vascular architecture with physiological force. Nature, 515(7526), 279-282. doi:10.1038/nature13701

Lindner M, Fokuhl J, Linsmeier F, Trebst C, Stangel M (2009) Chronic toxic demyelination in the central nervous system leads to axonal damage despite remyelination. Neurosci Lett. 453: 120-125. doi: 10.1016/j.neulet.2009.02.004

Louis, E. D., Ma, K., Babij, R., Cortes, E., Liem, R. K., Vonsattel, J. P., \& Faust, P. L. (2012). Neurofilament protein levels: quantitative analysis in essential tremor cerebellar cortex. Neurosci Lett, 518(1), 49-54. doi:10.1016/j.neulet.2012.04.054

Lourenco, T., \& Graos, M. (2016). Modulation of Oligodendrocyte Differentiation by Mechanotransduction. Front Cell Neurosci, 10, 277. doi:10.3389/fncel.2016.00277

Lourenco, T., Paes de Faria, J., Bippes, C. A., Maia, J., Lopes-da-Silva, J. A., Relvas, J. B., \& Graos, M. (2016). Modulation of oligodendrocyte differentiation and maturation by combined biochemical and mechanical cues. Sci Rep, 6, 21563. doi:10.1038/srep21563

McHugh, B. J., Buttery, R., Lad, Y., Banks, S., Haslett, C., \& Sethi, T. (2010). Integrin activation by Fam38A uses a novel mechanism of R-Ras targeting to the endoplasmic reticulum. J Cell Sci, 123(Pt 1), 51-61. doi:10.1242/jcs.056424

McHugh, B. J., Murdoch, A., Haslett, C., \& Sethi, T. (2012). Loss of the integrin-activating transmembrane protein Fam38A (Piezo1) promotes a switch to a reduced integrindependent mode of cell migration. PLoS One, 7(7), e40346. doi:10.1371/journal.pone.0040346

Misslin, C., Velasco-Estevez, M., Albert, M., O'Sullivan, S. A., \& Dev, K. K. (2017). Phospholipase A2 is involved in galactosylsphingosine-induced astrocyte toxicity, neuronal damage and demyelination. PLoS One, 12(11), e0187217. doi:10.1371/journal.pone. 0187217

O'Meara, R. W., Michalski, J. P., \& Kothary, R. (2011). Integrin signaling in oligodendrocytes and its importance in CNS myelination. J Signal Transduct, 2011, 354091. doi:10.1155/2011/354091

O'Sullivan, C., \& Dev, K. K. (2015). Galactosylsphingosine (psychosine)-induced demyelination is attenuated by sphingosine 1-phosphate signalling. J Cell Sci, 128(21), 3878-3887. doi: $10.1242 /$ jcs. 169342

O'Sullivan, S. A., Velasco-Estevez, M., \& Dev, K. K. (2017). Demyelination induced by oxidative stress is regulated by sphingosine 1-phosphate receptors. Glia, 65(7), 11191136. doi: $10.1002 /$ glia.23148

Ousman SS \& David S. (2000) Lysophosphatidylcholine induces rapid recruitment and activation of macrophages in the adult mouse spinal cord. Glia. 2000 Mar;30(1):92104. doi: 10.1002/(SICI)1098-1136(200003)30:1<92::AID-GLIA10>3.0.CO;2-W

Paez PM, Fulton D, Spreuer V, Handley V, Campagnoni AT. (2011). Modulation of canonical transient receptor potential channel 1 in the proliferation of oligodendrocyte precursor cells by the golli products of the myelin basic protein gene. J Neurosci. 31(10), 3625-37. doi: 10.1523/JNEUROSCI.4424-10.2011.

Pathak, M. M., Nourse, J. L., Tran, T., Hwe, J., Arulmoli, J., Le, D. T., . . Tombola, F. (2014). Stretch-activated ion channel Piezo1 directs lineage choice in human neural stem cells. 
Proc Natl Acad Sci U S A, 111(45), 16148-16153. doi:10.1073/pnas.1409802111

Plemel, J. R., Michaels, N. J., Weishaupt, N., Caprariello, A. V., Keough, M. B., Rogers, J. A., . . Yong, V. W. (2018). Mechanisms of lysophosphatidylcholine-induced demyelination: A primary lipid disrupting myelinopathy. Glia, 66(2), 327-347. doi:10.1002/glia.23245

Poole, K., Herget, R., Lapatsina, L., Ngo, H. D., \& Lewin, G. R. (2014). Tuning Piezo ion channels to detect molecular-scale movements relevant for fine touch. Nat Commun, 5, 3520. doi: $10.1038 /$ ncomms 4520

Redaelli E, Cassulini RR, Silva DF, Clement H, Schiavon E, Zamudio FZ, Odell G, Arcangeli A, Clare JJ, Alagón A, de la Vega RC, Possani LD, Wanke E. (2010). Target promiscuity and heterogeneous effects of tarantula venom peptides affecting $\mathrm{Na}+$ and K+ ion channels. J Biol Chem. 285(6), 4130-42. doi: 10.1074/jbc.M109.054718.

Rudrabhatla, P. (2014). Regulation of neuronal cytoskeletal protein phosphorylation in neurodegenerative diseases. J Alzheimers Dis, 41(3), 671-684. doi:10.3233/jad130794

Saab, A. S., Tzvetanova, I. D., \& Nave, K. A. (2013). The role of myelin and oligodendrocytes in axonal energy metabolism. Curr Opin Neurobiol, 23(6), 1065-1072. doi:10.1016/j.conb.2013.09.008

Shimizu, T., Osanai, Y., Tanaka, K. F., Abe, M., Natsume, R., Sakimura, K., \& Ikenaka, K. (2017). YAP functions as a mechanotransducer in oligodendrocyte morphogenesis and maturation. Glia, 65(2), 360-374. doi:10.1002/glia.23096

Simons M, Misgeld T, Kerschensteiner M (2014) A unified cell biological perspective on axonmyelin injury. J Cell Biol. 206: 335-345. doi: 10.1083/jcb.201404154

Simons, M., \& Nave, K. A. (2015). Oligodendrocytes: Myelination and Axonal Support. Cold Spring Harb Perspect Biol, 8(1), a020479. doi:10.1101/cshperspect.a020479

Sofroniew, M. V. (2009). Molecular dissection of reactive astrogliosis and glial scar formation. Trends Neurosci. 32(12), 638-47. doi: 10.1016/j.tins.2009.08.002.

Sofroniew, M. V., Vinters, H. V. (2010). Astrocytes: biology and pathology. Acta Neuropathol. 119(1), 7-35. doi: 10.1007/s00401-009-0619-8.

Stirling, D. P., Cummins, K., Wayne Chen, S. R., \& Stys, P. (2014). Axoplasmic reticulum $\mathrm{Ca}(2+)$ release causes secondary degeneration of spinal axons. Ann Neurol, 75(2), 220-229. doi:10.1002/ana.24099

Su, Z., Yuan, Y., Chen, J., Cao, L., Zhu, Y., Gao, L., . . He, C. (2009). Reactive astrocytes in glial scar attract olfactory ensheathing cells migration by secreted TNF-alpha in spinal cord lesion of rat. PLoS One, 4(12), e8141. doi:10.1371/journal.pone.0008141

Suchyna, T. M., Johnson, J. H., Hamer, K., Leykam, J. F., Gage, D. A., Clemo, H. F., . . Sachs, F. (2000). Identification of a peptide toxin from Grammostola spatulata spider venom that blocks cation-selective stretch-activated channels. J Gen Physiol, 115(5), 583598. doi: 10.1085/jgp.115.5.583

Syeda, R., Xu, J., Dubin, A. E., Coste, B., Mathur, J., Huynh, T., . . Patapoutian, A. (2015). Chemical activation of the mechanotransduction channel Piezo1. Elife, 4, e07369. doi:10.7554/eLife.07369

Velasco-Estevez, M., Mampay, M., Boutin, H., Chaney, A., Warn, P., Burgess, E., ... Sheridan, G. K. (2018). Infection augments expression of mechanosensing Piezol channels in amyloid plaque-reactive astrocytes. Front Aging Neurosci. 10: 332. doi:10.3389/fnagi.2018.00332

Velasco-Estevez, M., Rolle, S.O., Mampay, M., Dev, KK., \& Sheridan, G.K. (2019) Piezo1 regulates calcium oscillations and cytokine release from astrocytes. Glia, Accepted (In Press) doi: 10.1002/glia.23148

Wang, J., Ma, Y., Sachs, F., Li, J., \& Suchyna, T. M. (2016). GsMTx4-D is a cardioprotectant against myocardial infarction during ischemia and reperfusion. $J \mathrm{Mol}$ Cell Cardiol, 98, 83-94. doi:10.1016/j.yjmcc.2016.07.005

Wang W, Gao XF, Xiao L, Xiang ZH, He C. (2011) K(V)7/KCNQ channels are functionally expressed in oligodendrocyte progenitor cells. PLoS One. 6(7), e21792. doi:10.1371/journal.pone.0021792.

Wang, Y. Y., Zhang, H., Ma, T., Lu, Y., Xie, H. Y., Wang, W., Ma, Y. H., Li, G. H., \& Li, Y. W. (2019) Piezo1 mediates neuron oxygen-glucose deprivation/reoxygenation injury via 
$\mathrm{Ca}^{2+} /$ calpain signaling. Biochem Biophys Res Commun. 513: 147-153. doi:10.1016/j.bbrc.2019.03.163

Ward, C. W., Sachs, F., Bush, E. D., \& Suchyna, T. M. (2018). GsMTx4-D provides protection to the D2.mdx mouse. Neuromuscul Disord, 28(10), 868-877. doi:10.1016/j.nmd.2018.07.005

Wojda, U., Salinska, E., \& Kuznicki, J. (2008). Calcium ions in neuronal degeneration. IUBMB Life, 60(9), 575-590. doi:10.1002/iub.91

Yeung, E. W., Whitehead, N. P., Suchyna, T. M., Gottlieb, P. A., Sachs, F., \& Allen, D. G. (2005). Effects of stretch-activated channel blockers on $[\mathrm{Ca} 2+] \mathrm{i}$ and muscle damage in the mdx mouse. J Physiol, 562(Pt 2), 367-380. doi:10.1113/jphysiol.2004.075275

Yuan A, Rao MV, Veeranna, Nixon RA (2017). Neurofilaments and Neurofilament Proteins in Health and Disease. Cold Spring Harb Perspect Biol. 9: a018309. doi: 10.1101/cshperspect.a018309

Zhao, Q., Zhou, H., Li, X., \& Xiao, B. (2018). The mechanosensitive Piezo1 channel: a threebladed propeller-like structure and a lever-like mechanogating mechanism. Febs $J$. 286(13), 2461-2470. doi:10.1111/febs.14711

Zundorf, G., \& Reiser, G. (2011). Calcium dysregulation and homeostasis of neural calcium in the molecular mechanisms of neurodegenerative diseases provide multiple targets for neuroprotection. Antioxid Redox Signal, 14(7), 1275-1288. doi:10.1089/ars.2010.3359 